\title{
Expression pattern of glycoside hydrolase genes in Lutzomyia longipalpis reveals key enzymes involved in larval digestion
}

\section{Caroline da Silva Moraes ${ }^{1+}{ }^{+}$Hector M. Diaz-Albiter ${ }^{1}$, Maiara do Valle Faria ${ }^{1}$, Maurício R. V. Sant'Anna ${ }^{2,3}$, Rod J. Dillon ${ }^{3}$ and Fernando A. Genta ${ }^{1,4 *}$}

\footnotetext{
${ }^{1}$ Laboratory of Insect Biochemistry and Physiology, Department of Biochemistry and Molecular Biology, Oswaldo Cruz Institute, FIOCRUZ, Rio de Janeiro, Brazil

2 Parasitology Department, Federal University of Minas Gerais, Belo Horizonte, Brazil

${ }^{3}$ Faculty of Health and Medicine, Division of Biomedical and Life Sciences, Lancaster University, Lancaster, UK

${ }^{4}$ National Institute of Science and Technology, Department of Molecular Entomology, Laboratory of Insect Biochemistry and Physiology, Rio de Janeiro, Brazil
}

Edited by:

Raman Chandrasekar, Kansas State University, USA

Reviewed by:

Raman Chandrasekar, Kansas State University, USA

Manickam Sugumaran, University of

Massachusetts Boston, USA

\section{*Correspondence:}

Fernando A. Genta, Laboratório de Bioquímica e Fisiologia de Insetos, Instituto Oswaldo Cruz, FIOCRUZ, Av. Brasil 4365, Pavilhão Leônidas Deane, Sala 207. Manguinhos, Rio de Janeiro, CEP 21040-360, Brazil e-mail:genta@ioc.fiocruz.br

\section{${ }^{\dagger}$ Present Address:}

Caroline da Silva Moraes, Centro Universitário Augusto Motta, Rio de Janeiro, Brazil
The sand fly Lutzomyia longipalpis is the most important vector of American Visceral Leishmaniasis. Adults are phytophagous (males and females) or blood feeders (females only), and larvae feed on solid detritus. Digestion in sand fly larvae has scarcely been studied, but some glycosidase activities putatively involved in microorganism digestion were already described. Nevertheless, the molecular nature of these enzymes, as the corresponding genes and transcripts, were not explored yet. Catabolism of microbial carbohydrates in insects generally involves $\beta-1,3-$ glucanases, chitinases, and digestive lysozymes. In this work, the transcripts of digestive $\beta-1,3-$ glucanase and chitinases were identified in the L. longipalpis larvae throughout analysis of sequences and expression patterns of glycoside hydrolases families 16,18 , and 22 . The activity of one i-type lysozyme was also registered. Interestingly, this lysozyme seems to play a role in immunity, rather than digestion. This is the first attempt to identify the molecular nature of sand fly larval digestive enzymes.

Keywords: Lutzomyia longipalpis, digestion, $\beta$-1,3-glucanase, chitinase, lysozyme

\section{INTRODUCTION}

Phlebotomines are psychodid dipterans distributed over almost all faunal regions of the world and particularly abundant in temperate and inter-tropical regions. There are more than 600 described species to date. However, only 30 of these are epidemiologically important for pathogen transmission. Phlebotomine sand flies are able to transmit several diseases such as Leishmaniasis, which occur in approximately 98 countries and affects millions of people each year (WHO, 2014), as well as Bartonellosis (Tsai et al., 2011) and arbovirosis (Amaro et al., 2012).

Leishmaniasis are zoonosis caused by infection with Leishmania genus parasites and these are divided clinically in three forms (cutaneous, mucocutaneous, and visceral) depending on the parasite species. In the New World, Visceral Leishmaniasis is a severe systemic disease caused by Leishmania infantum (syn. L. chagasi) and transmitted by phlebotomines of the species Lutzomyia longipalpis (Romero and Boelaert, 2010; Harhay et al., 2011).

Like other Diptera, phlebotomines are holometabolous insects with adult and larval stages which exploit different food sources. Male and female adults feed on plant sap (or blood, in the case of females) (Brazil and Brazil, 2003; Soares and Turco, 2003) while larvae grow on decaying organic matter, mainly of plant origin, or animal feces. Wermelinger and Zanuncio (2001) found that $L$. longipalpis and $L$. intermedia larvae improved development until pupation when fed a variety of the diet described by Young et al. (1981), constituted by humus containing an intense proliferation of fungi. The authors suggested that this composition mimicked the larval substrate in the wilderness. However, despite these observations in the laboratory, little is known about the natural habitat of phlebotomine larvae (Feliciangeli, 2004).

Differences between larval and adult feeding habits of sand flies correlate with anatomical, physiological, and biochemical variations within the phlebotomine digestive tract. Therefore, it is not accurate to generalize about digestion throughout the development of these insects. Although some authors have shown the presence of both proteases and carbohydrases in sand flies (Dillon and El Kordy, 1997; Gontijo et al., 1998; Jacobson and Schlein, 2001; Ramalho-Ortigão and Traub-Csekö, 2003; Do Vale et al., 2007; Telleria et al., 2007, 2010; Sant'Anna et al., 2009), the role of digestible carbohydrases in phlebotomines and their potential participation in the digestion of microorganisms has not been systematically studied.

Moraes et al. (2012) described several glycosidases in larvae of L. longipalpis which may be related to the digestion of bacteria and fungi. This suggested that sand fly larvae have detritivorous feeding habits. The authors also assayed enzymatic activity 
of $\beta$-glucanases, chitinases, and lysozymes and confirmed that $\beta$-1,3-glucanases exhibited the highest specific activities in the luminal contents of the larval gut.

$\beta$-1,3-glucanases ( $\beta \mathrm{Glu})$ are glycoside hydrolases $(\mathrm{GH})$ responsible for the hydrolysis of $\beta$-1,3-glucans which are found in the cell walls of fungi (Bartnicki-Garcia, 1968; Gorin and Spencer, 1968; Sietsma and Wessels, 1981) and phloem of higher plants as callose (Bacic et al., 1998). These enzymes are commonly found in the gut and salivary glands of insects such as Spodoptera frugiperda (Bragatto et al., 2010), Tenebrio molitor (Genta et al., 2009), and Periplaneta americana (Genta et al., 2003).

Besides $\beta$-1,3-glucanases, insects also express $\beta$-glucan recognition proteins ( $\beta$ GRPs), $\beta$-glucan binding proteins (GBP) and gram-negative bacteria binding proteins (GNBP) responsible for triggering the innate immune response through recognition of pathogen-associated molecular proteins (PAMPs) such as lipopolysaccharide (LPS), peptidoglycan, and $\beta$-1,3-glucan (Royet, 2004). These proteins are found mainly in hemolymph and fat body of insects (Ma and Kanost, 2000; Wang et al., 2005; Sun et al., 2011). Both $\beta$-1, 3-glucanases and $\beta$-glucan-binding proteins found in insects have been structurally assigned to family 16 of glycoside hydrolases (GH16) (Genta et al., 2009; Bragatto et al., 2010).

Chitinases (Chit) are enzymes able to hydrolyze chitin, a linear polymer of $\mathrm{N}$-acetylglucosamine (GlcNAc) present in the cell wall of fungi as well as in insect structures such as cuticle, trachea, peritrophic matrix, and gut (Arakane and Muthukrishnan, 2010). All insect chitinases belong to family 18 of glycoside hydrolases (GH18) and are responsible for cuticle turnover, as well as digestion and degradation of the peritrophic matrix during molting (Zhang et al., 2011). GH18 are encoded by several groups of genes which are constituted by a multi-domain structural organization that includes: (1) 1-5 catalytic domains; (2) 0-7 cysteine-rich chitin-binding domains (CBD), and (3) serine/threonine-rich linker regions that can be heavily glycosylated (Merzendorfer and Zimoch, 2003; Arakane and Muthukrishnan, 2010).

Chitinases have been studied in different insect orders, in species such as Anopheles gambiae (Shen and Jacobs-Lorena, 1997; Zhang et al., 2011), Aedes aegypti (De la Vega et al., 1998), Manduca sexta (Kramer et al., 1993), Bombyx mori (Kim et al., 1998; Zhang et al., 2011), Hyphantria cunea (Kim et al., 1998), Drosophila melanogaster (Zhu et al., 2008), Ostrinia nubilalis (Khajuria et al., 2010), Tribolium castaneum (Zhu et al., 2008), Tenebrio molitor (Royer et al., 2002; Genta et al., 2006), and Phlebotomus papatasi (Ramalho-Ortigão et al., 2005; CoutinhoAbreu et al., 2010). Ramalho-Ortigão and Traub-Csekö (2003) isolated and characterized cDNA encoding a chitinase from the gut tissue of adult female L. longipalpis (Llchit1), which seems to be involved in the degradation of the peritrophic membranes and Leishmania migration toward the midgut epithelium during blood digestion.

Lysozymes (Lys) are glycoside hydrolases belonging to the glycoside hydrolases family 22 (GH22), whose function is to catalyze hydrolysis of glycosidic bonds between $\mathrm{N}$-acetylmuramic acid (NAM) and N-acetylglucosamine (NAG). These molecules are constitutive components of the peptidoglycan layer of bacterial cell walls (Jollès and Jollès, 1984). Lysozymes are widely found in various organisms and are divided into six major groups. C-type lysozymes are probably the best studied and are found in several vertebrate and invertebrate taxa, including the vast class Insecta (Bachali et al., 2002; Harikrishnan et al., 2011). In addition to c-type lysozymes, other lysozymes have been studied, such as itype lysozymes. This new class of lysozymes shows similarity with destabilases found in annelids (Zavalova et al., 2000), molluscs (Ito et al., 1999), and insects (Paskewitz et al., 2008). Zavalova et al. (2000) showed activity of i-type lysozyme in the cell wall of Micrococcus lysodeikticus and inhibition of such activity by antidestabilase. However, Paskewitz et al. (2008) found no activity of i-type lysozyme in Anopheles gambiae.

An important hindrance for the study of sand fly enzymes is the diminute size of these animals, and their laborious and time-consuming maintenance of colonies, which hardly affords samples with amounts of protein enough for activity screenings. For this reason, the traditional strategies of enzyme purification, characterization, and sequencing, which were used in the study of other insect digestive glucanases and chitinases (Genta et al., 2009), are not applicable to this insect model. Anterior work from our group (Moraes et al., 2012) showed the presence of $\beta$-1,3-glucanase, chitinase, and lysozyme activities in the gut of sand fly larvae, but there are no description of any protein or DNA sequence related to digestive enzymes in these insects. In this work, we studied the expression pattern of different genes from families GH16, GH18, and GH22 in L. longipalpis and correlated two of them to the digestion of larvae. To our knowledge, this is the first report on the molecular identification of digestive enzymes in phlebotomine larvae.

\section{MATERIALS AND METHODS SAND FLY REARING AND LARVAE FEEDING}

Insects used in all experiments were insectary-reared L. longipalpis from a colony originally started from individuals from Jacobina, Brazil, and maintained at the Laboratory of Insect Biochemistry and Physiology (Oswaldo Cruz Institute, FIOCRUZ). Adult sandflies were fed a $70 \%$ sucrose solution (w/v) ad libitum. Adult females were blood-fed with anesthetized hamsters (ketamine, $200 \mathrm{mg} / \mathrm{kg}$ ) to trigger egg development. After oviposition, eggs were collected and reared to preserve the colony. All larval instars were fed a crushed mixture of rabbit feces, rabbit chow, and garden soil. Third and fourth instars were supplemented with a mixture of white soy protein (bran) and cereal flakes (Neston) (1:1).

\section{IN SILICO MINING OF L. LONGIPALPIS ESTS LIBRARY FOR GLYCOSIDE HYDROLASES}

To identify sequences of families 16 ( $\beta-1,3-$ Glucanases, GBP), 18 (chitinases), and 22 (lysozymes), glycoside hydrolases from different insect orders were identified and retrieved from CAZy (Carbohydrate-Active Enzyme database, http://www.cazy.org/). Subsequently, GH sequences obtained from CAZy were employed to perform a TBLASTX search (http://blast.ncbi.nlm.nih.gov/) to find similar sequences in a Lu. longipalpis EST library (Dillon et al., 2006) at the Sanger Institute website (http://www.genedb. org/Page/parasiteVectors). 
ANALYSIS OF GH SEQUENCES FROM THE L. LONGIPALPIS EST LIBRARY Sequences retrieved from the L. longipalpis EST library were translated using the Translate tool (http://web.expasy.org/ translate/) and compared against the non-redundant protein database from the National Center for Biotechnology Information (NCBI). Analysis of Lu. longipalpis ESTs similarity to GH families 16, 18, and 22 as well as prediction of ORF integrity was performed using the BLASTP tool. Translated sequences were further analyzed to identify signal peptides, O-type glycosylation, $\mathrm{N}$-type glycosylation, and functional domains using PeptideIP Server 4.0 (Petersen et al., 2011; http://www.cbs.dtu.dk/services/ SignalP/), NetOGlyc Server 3.1 (Julenius et al., 2005; http://www. cbs.dtu.dk/services/NetOGlyc/), NetNGlyc Server 1.0 (http:// www.cbs.dtu.dk/services/NetNGlyc/), and database PFAM 26.0 (Punta et al., 2012; http://pfam.sanger.ac.uk/), respectively. Alignments were performed using ClustalW (http://ebi.ac.uk/ Tools/msa/clustalw2/) (Hall, 1999).

\section{PHYLOGENETIC ANALYSIS OF L. LONGIPALPIS GH16, GH18, AND GH22}

Neighbor-joining phylogenetic trees for each gene family were constructed using insect protein sequences with PFAM domains GH16_beta_GRP (CD02179, $\beta$-1,3-glucanases and $\beta$-glucan binding proteins), Glyco_hydro_18 (PF00704, chitinases), and LYS (PF00062) and Destabilase (CD05497) for lysozymes. Trees were generated using MEGA5.05 (Tamura et al., 2011). Bootstrap values were set at 5000 replications.

\section{DISSECTIONS}

4th instar larvae of $L u$. longipalpis were rinsed in $200 \mu \mathrm{L}$ of sterile $0.15 \mathrm{M} \mathrm{NaCl}$ solutions and anesthetized on ice. Larvae were dissected and the following structures removed: head (including foregut), midgut and hindgut $(\mathrm{M}+\mathrm{H})$ and carcass (rest of the body). Matching tissues were pooled in groups of 5 and then transferred to polypropylene vials containing $50 \mu \mathrm{L}$ of TRI Reagent ${ }^{\circledR}$ (Sigma). Samples were flash-frozen and kept at $-80^{\circ} \mathrm{C}$ until further RNA extraction.

\section{RNA EXTRACTION AND cDNA SYNTHESIS}

RNA was extracted from entire insects, head, $\mathrm{M}+\mathrm{H}$ and carcass tissue samples, according to Diaz-Albiter et al. (2011). After extraction, total RNA was quantified using Nanodrop ${ }^{\circledR}$ (NanoDrop Technologies, Wilmington, USA). RNA was reversetranscribed to cDNA using SuperScript III First-Strand Synthesis System (Invitrogen, San Diego, CA) following the manufacturer's protocol. cDNA was quantitated using Nanodrop and normalized to a concentration of $50 \mathrm{ng} / \mu \mathrm{l}$.

\section{TISSUE-SPECIFIC EXPRESSION OF $\beta$ GLU, GBP, Chit, AND Lys}

Polymerase Chain Reaction (PCR) and multiplex PCR were used to assess tissue-specific expression of $\beta$-Glu and GBP, Chit, and Lys in fourth instar larvae. For multiplex PCR, three specific primers were included in the same reaction, which allowed simultaneous amplification of more than one gene. Primer combinations were as follows: (1) 11b04, LamS2, 24g06, and Ribo60; (2) $96 \mathrm{~h} 07$ and 154b12; and (3) 123b01 and 18f06. For sequences $88 \mathrm{~d} 12$ and $14 \mathrm{~b} 06$, PCRs were performed using only a pair of primers. All primers have listed in Table S1 in Supplementary
Material. Amplification reactions were performed in a total volume of $20 \mu \mathrm{L}$ containing $50 \mathrm{ng} \mathrm{cDNA}, 0.5 \mu \mathrm{M}$ of each primer, $1 \times \mathrm{PCR}$ reaction buffer, $0.2 \mathrm{mM}$ each $\mathrm{dNTP}, 1.5 \mathrm{mM}$ magnesium chloride and $0.025 \mathrm{U}$ GoTaq ${ }^{\circledR}$ DNA polymerase (Promega). The parameters for PCR were: incubation at $94^{\circ} \mathrm{C}$ for $2 \mathrm{~min}$ followed by varying cycles of $94^{\circ} \mathrm{C}$ for $15 \mathrm{~s}, 55^{\circ} \mathrm{C}$ for $30 \mathrm{~s}, 72^{\circ} \mathrm{C}$ for $1 \mathrm{~min}$ and a final incubation (extension) of $72^{\circ} \mathrm{C}$ for $5 \mathrm{~min}$. The number of cycles vary depending on the combination of primers used in the PCR and PCR-Multiplex reactions, as shown in Table S2 in the Supplementary Material. PCR products were analyzed by gel electrophoresis using agarose $2 \%(\mathrm{w} / \mathrm{v})$, stained with ethidium bromide $(0.5 \mu \mathrm{g} / \mathrm{mL})$. Expression patterns were obtained by measuring the band intensity by densitometry using ImageJ software and then calculating relative expressions against a constitutive gene (AM088777, 60S ribosomal protein L3).

\section{STATISTICAL ANALYSIS}

All statistical analyses were performed using GraphPad Prism 5.0 for Windows, (San Diego California USA). Student's $t$-test for paired data was used. Comparison among and between groups was assessed using One-Way analysis of variance (ANOVA) with a post-hoc Tukey multiple comparison test. Results are expressed as the group mean \pm s.e.m. Significance was considered when $p<0.05$.

\section{RESULTS}

\section{IN SILICO ANALYSIS OF GHS SEOUENCES RETRIEVED FROM THE L. LONGIPALPIS EST LIBRARY}

A total of 206 sequences of glycoside hydrolases families 16, 18 , and 22 from different insect species were found in CAZy, 85 of which belonged to family GH16, 47 to GH18, and 74 to GH22. They were employed to perform an in silico search targeting these three protein families in an L. longipalpis ESTs library (http://www.genedb.org/Page/parasiteVectors, Dillon et al., 2006). A total of 9 sequences were retrieved, 3 corresponding to clones of GH16 (identifiers: NSFM-140g04, NSFM-14b06, and NSFM-111b04), 5 corresponding to GH18 (identifiers: NSFM-18f06, NSFM-88d12, NSFM-24g06, NSFM96h07, and NSFM-154b12) and 1 corresponding to GH22 (identifier: NSFM-123b01). Only five out of nine sequences displayed non-truncated open read frames (ORFs): NSFM-140g04 and NSFM-14b04 (GH16), NSFM-18f06 and NSFM-154b12 (GH18), and NSFM-123b01 (GH22). Moreover, prediction of signal peptides in these sequences suggests that their putative proteins are secreted. According to our analysis, hypothetical complete GH proteins have predicted molecular masses of 40-41, 43-47, and $15 \mathrm{kDa}$ and estimated isoelectric points of $6.2-7.8,6.8-8.4$, and 4.9 (GHF16, GHF18, and GHF22, respectively).

Sequences of interest found in the L. longipalpis EST library were BLASTed against NCBI-NR and identified according to their best hit. All analyzed sequences were identified as proteins belonging to families GH16, GH18, and GH22, as shown in Table S3.

Amino acid sequences alignments of $L$. longipalpis proteins from GHF16, GHF18, and GHF22 with members from different insect groups exhibited highly-conserved regions (in Supplementary Material). L. longipalpis GH16 sequence of clone 
NSFM-140g04 was constituted by 385 amino acid sequence and highly-conserved regions when compared to other insect $\beta$-1,3glucanases. These conserved regions also include typical family GH16 catalytic glutamate residues with proton donor-acceptor functions and are located at positions 196 and 201. Also, the sequence has a putative signal peptide with cleavage sites at positions 19 and 20 (Figure S1).

Putative $\beta$-glucan binding protein clones NSFM-111b01 and NSFM-14b06 found in the L. longipalpis EST library were constituted by 268 and 370 amino acid residues, respectively. These sequences shared similar regions with insect $\beta$-glucan binding proteins, also known as Gram-negative-binding proteins (GNBPs), (Figure S2). Since NSFM-111b01 was a truncated sequence at the $5^{\prime}$ region, it was not possible to evaluate the presence of a signal peptide. On the other hand, NSFM$14 \mathrm{~b} 06$ did display a signal peptide with cleavage sites between positions 17 and 18. Interestingly, NSFM-111b01 and NSFM$14 \mathrm{~b} 06$ lacked the catalytic glutamate residues responsible for hydrolysis of $\beta$-1,3-glucan otherwise present in $\beta$-1,3-glucanases (Figure S2).

Alignment of family GH18-like sequences showed that clones NSFM-154b12, NSFM-88d12, and NSFM-18f06 had a high level of conservation with other insect chitinases. Results of chitinases and chitinases-like domain organization analysis are summarized in Figure S3.

Clone NSFM-96h07 consisted of a small 113 amino acid region located in the protein catalytic domain. It was not possible to verify the presence of a signal peptide because $3^{\prime}$ and $5^{\prime}$ ORFs regions were truncated.

Insect chitinases share four different conserved regions (CR1 to CR4). Clone NSFM-154b12 consisted of 392 amino acids and showed a putative signal peptide with cleavage sites between amino acids 19 and 20. This sequence shared similarity in CR2 and CR3 (Figure S4). However, CR1 and CR4 were not found in this sequence. Clone NSFM-88d 12 consisted of 296 amino acids and was truncated at region $5^{\prime}$, lacking a signal peptide and CR1. On the other hand, CR2, CR3, and CR4 were present and CR2 displayed the catalytic glutamate (E40) (Figure S5).

NSFM-24g06 consisted of 201 amino acid residues. The alignment of this sequence with other insect chitinases allowed identification of CR3 and also suggests that NSFM-24g06 is incomplete (Figure S6). The presence of CR1 and CR2 and the presence of catalytic residues could not be evaluated.

Clone NSFM-96h07 showed low conservation in amino acid sequence in CR1 and CR2 and no evidence of CR3 and CR4. Furthermore, NSFM-96h07 displayed a substitution of glutamate (E) with glutamine $(\mathrm{Q} 75)$ at the hypothetical catalytic residue (Figure S7).

NSFM-18f06 consisted of 441 residues. This sequence showed a signal peptide with cleavage sites between amino acids 21 and 22. CR1 and CR2 were identified within this sequence. A serine residue (S152) was identified in CR2, unlike the all other sequence analyzed which had a glycine and alanine residue at that position. CR3 and CR4 were not identified in the alignment (Figure S8).

Lysozyme-like clone NSFM-123b01 consisted of 165 amino acid residues and appeared to be complete as suggested by the presence of a methionine residue and a stop codon. Furthermore, SignalIP showed the presence of signal peptide cleavage sites between amino acids 22 and 23, (Figure S9).

\section{PHYLOGENETIC ANALYSIS OF GH16, GH18, AND GH22 SEOUENCES FOUND IN THE L. LONGIPALPIS ESTS LIBRARY}

To classify all L. longipapis genes found in families GH16, GH18, and $\mathrm{GH} 22$ and to predict a physiological role of these putative enzymes, phylogenetic trees were constructed using a Neighborjoining algorithm. Subgroups considered for phylogenetic analysis were $\beta$-glucanases and $\beta$-glucan binding proteins for family GH16, I to VIII-chitinase groups of families GH18 (Zhang et al., 2011), and i and c-type lysozymes for family GH22 (Bachali et al., 2002; Paskewitz et al., 2008). Analysis of GH16 was restricted to sequences from order Diptera while in GH18 and GH22 it considered several orders of insects.

Phylogenetic analysis of family GH16 revealed that sequences found in the L. longipalpis ESTs library separately groups with each of the two major clades found in this family: one clade includes sequences with high bootstrap, all lacking the catalytic glutamates (e.g., NSFM-111b04), and other clade which includes sequences with low bootstrap and catalytic residues (e.g., NSFM140g04) (Figure 1A). Moreover, the latter clade forms a paraphyletic group with a $C$. quinquefasciatus sequence annotated in GenBank as a Gram-negative bacteria binding protein, which harbors the glutamate catalytic residues.

There was no evidence of any Pfam domain for NSFM-14b06. Furthermore, NSFM-111b01 and NSFM-14b06 sequences lacked the catalytic site responsible for hydrolysis of $\beta$-1,3-glucan otherwise present in $\beta$-1,3-glucanases (Figure 1B). Taking into account the presence and location of a catalytic site in the sequences within the clade of digestive enzymes, we named NSFM-140g04 as L1 $\beta$ Glu, whereas NSFM-111b04 and NSFM-14b06 were named LlGBP1 and LlGBP2, respectively.

Phylogenetic analysis of GH18 divided 4 L. longipalpis chitinases into different clades. To perform this, we extracted the GH18 chitinase catalytic conserved domains (Pfam 00704), except for the NSFM-96h07 sequence which did not contain such domain.

In our analysis, chitinase subgroups I, II, III, V, VI, VII, and VIII formed monophyletic groups, while group IV formed a paraphyletic group (Figure 2). According to clade division, L. longipalpis sequences were divided as follows: NSFM-154b12 into type VIII, NSFM-88d12 into type II, NSFM-24g06 into type IV, and NSFM-18f06 into type V (Figure 2). After the chitinases classification from our filogenetics analysis, putative chitinases were named as follows: NSFM-154b12 is LlChit2, NSFM-88d12 is LlChit3, NSFM-24g06 is LlChit4, NSFM- 96h07 is LlChit5, and NSFM-18f06 is LlIDGF. The name LlChit1 was not used in this work to avoid confusion with the sequence described by Ramalho-Ortigão et al. (2005; LlChi1).

The phylogenetic tree of lysozymes from insects was divided in two clades: c-type lysozyme and i-type lysozymes, forming monophyletics groups in each clade (Figure 3). Positioning of the NSFM-123b01 sequence showed that this sequence belonging to the i-type lysozyme family (Figure 3), named in this paper as LlLysi. 


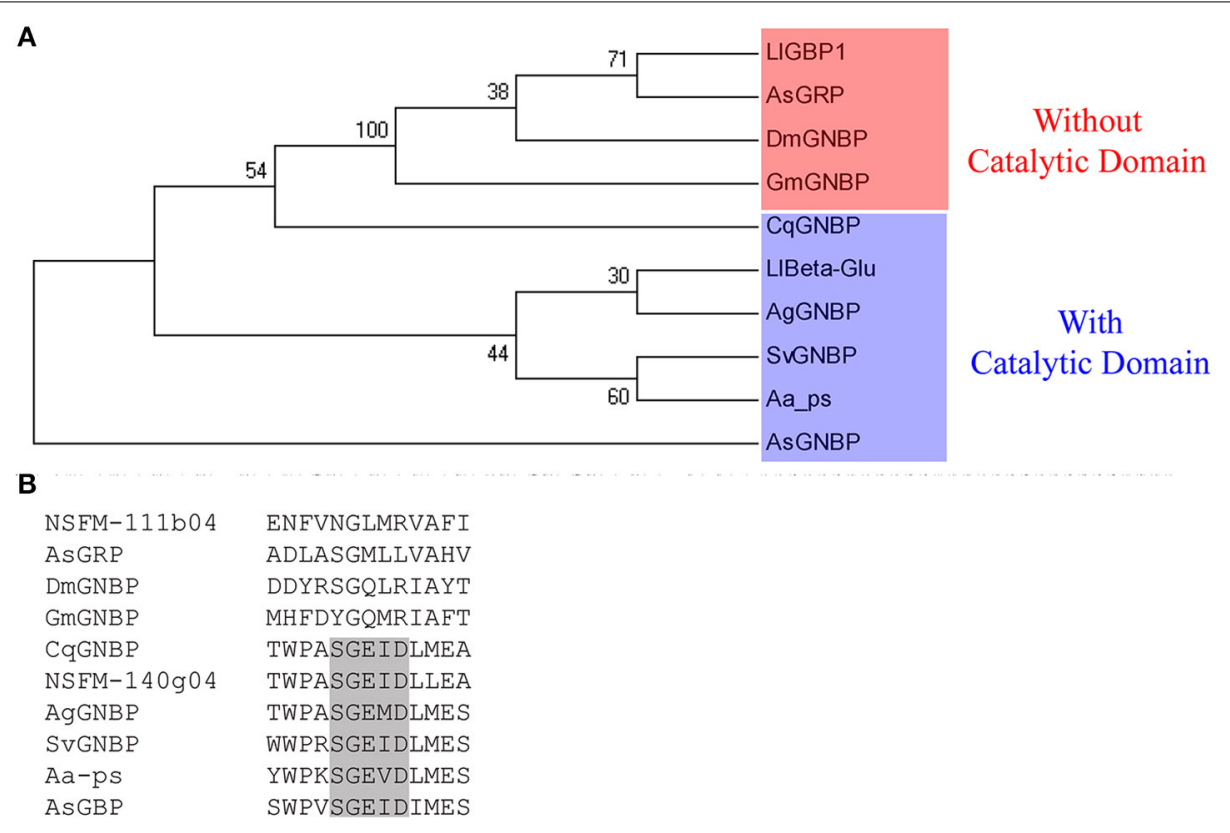

FIGURE 1 | Cladogram of selected GH16 proteins from the order Diptera. (A) Phylogenetic tree was done using sequences from Armigeres subalbatus (accession number AAT99011), Glossina morsitans morsitans (ABC25063), Drosophila melanogaster (AAF33851), Anopheles gambiae (ACN38130), Culex quinquefasciatus (AEQ27734), and Simulium vittatum (ACH56895). Actinophrys sol (Porifera, BAG32349) was used as outgroup. The sequences

\section{EXPRESSION OF $\beta$-1,3-GLUCANASES, CHITINASES, AND LYSOZYME DURING DEVELOPMENT OF L. LONGIPALPIS}

The expression of putative glycoside hydrolase-coding transcripts from families 16,18 , and 22 ( $\beta$-glucanases/ $\beta$-glucan binding proteins, chitinases, and lysozyme, respectively) was evaluated at different development stages and dietary conditions of L. longipalpis, namely larvae (L4), pupae (P), unfed male adults (UM), sugar-fed male adults (SM), unfed female adults (UF), sugar-fed female adults (SF), and blood-fed female adults (BF). Among GH16 sequences, Ll $\beta$ Glu showed a significantly higher relative expression in the L4 larval stages $(0.40 \pm 0.09)$ when compared to the other stages of development $(p<0.05)$ (Figure $4 A$ ). In contrast, binding proteins were similarly expressed throughout all stages (LlGBP1, Figure 4B) or poorly expressed in the larval stage (LlGBP2, Figure 4C).

LlGBP1 showed a higher relative expression in $\mathrm{P}(0.89 \pm$ $0.05)$, SM $(0.74 \pm 0.01)$, and BF $(0.7 \pm 0.1)$. Relative expression was significantly higher in $\mathrm{P}$ compared to L4 $(p<0.005)$, and UF and SF $(p<0.001)$. Furthermore, the relative expression of SM was significantly different compared with UF and SF $(p<0.0001)$. Expression in blood-fed females was statistically difference compared to SF $(p<0.05)$ (Figure 4B).

LlGBP2 showed higher relative expressions in the adult stage, specially in UM (3.4 \pm 0.5$), \mathrm{SM}(4.7 \pm 0.7)$, and BF (3.9 $\pm 0.5)$. However, expression in UM was only significantly different from L4 $(p<0.05)$, SM expression values were significantly different to L4 $(p<0.01)$ and $\mathrm{P}(p<0.05)$, while values in BF were different from L4 $(p<0.01)$ and $\mathrm{P}(p<0.01)$ (Figure 4C).
NSFM-111b04 and NSFM-140g04 retrieved from Lutzomyia longipalpis EST are named as LIGBP1 and LIBeta-Glu, respectively. Bootstrap values were obtained by neighbor-joining method (software MEGA 5.05) using 5000 replications. (B) Aligment of the $\beta-1,3$-glucanases active site sequences used for phylogenetic analysis showing the presence of the catalytic region (black box).

Chitinases-like transcripts also showed different expression patterns throughout the sand fly developmental stages. LlChit2 showed no significant differences between experimental groups (Figure 5A).

LlChit3 showed higher relative expression in L4 (1.8 \pm $0.1), \mathrm{P}(2.7 \pm 0.6)$ and $\mathrm{UM}(2.6 \pm 0.6)$. Values were significantly different when compared to SM $(p<0.01)$, while values in $\mathrm{P}$ were different from SM and UF, SF, and BF $(p<$ 0.05 ) (Figure 5B). LlChit4 showed high relative expression levels in L4 (2.8 \pm 0.1$)$ and SM (2.6 \pm 0.4$)$. Expression levels in L4 were significantly different from UF, SF, and BF $(p<$ $0.001)$. The sugar-fed males showed significant differences compared to all adult female groups UF, SF, and BF $(p<0.05)$ (Figure 5C).

LlChit5 showed higher relative expression in $\mathrm{P}(3.1 \pm 0.3)$ compared to all groups $(p<0.05)$ except UM (Figure 5D). LIIDGF showed significant differences in relative expression in SM $(16 \pm 9)$ compared to groups L4, P, UF, SF, and BF $(p<0.05)$ (Figure 5E).

LlLysi showed higher relative expression values UM (5 \pm 2 ). However, significant differences were only observed in L4 and BF $(p<0.05)$, as shown in Figure 6.

\section{TISSUE-SPECIFIC EXPRESSION OF $\beta$-1,3-GLUCANASES, CHITINASES, AND LYSOZYME IN LARVAE OF L. LONGIPALPIS}

Ll$\beta$ Glu showed a significantly higher relative expression level in larval guts when compared to other tissues and it appears to be gut-specific $(p<0.01$, Figure 7A). On the other hand, expression of LlGBP1 was significantly higher in carcass (Figure 7B) and 


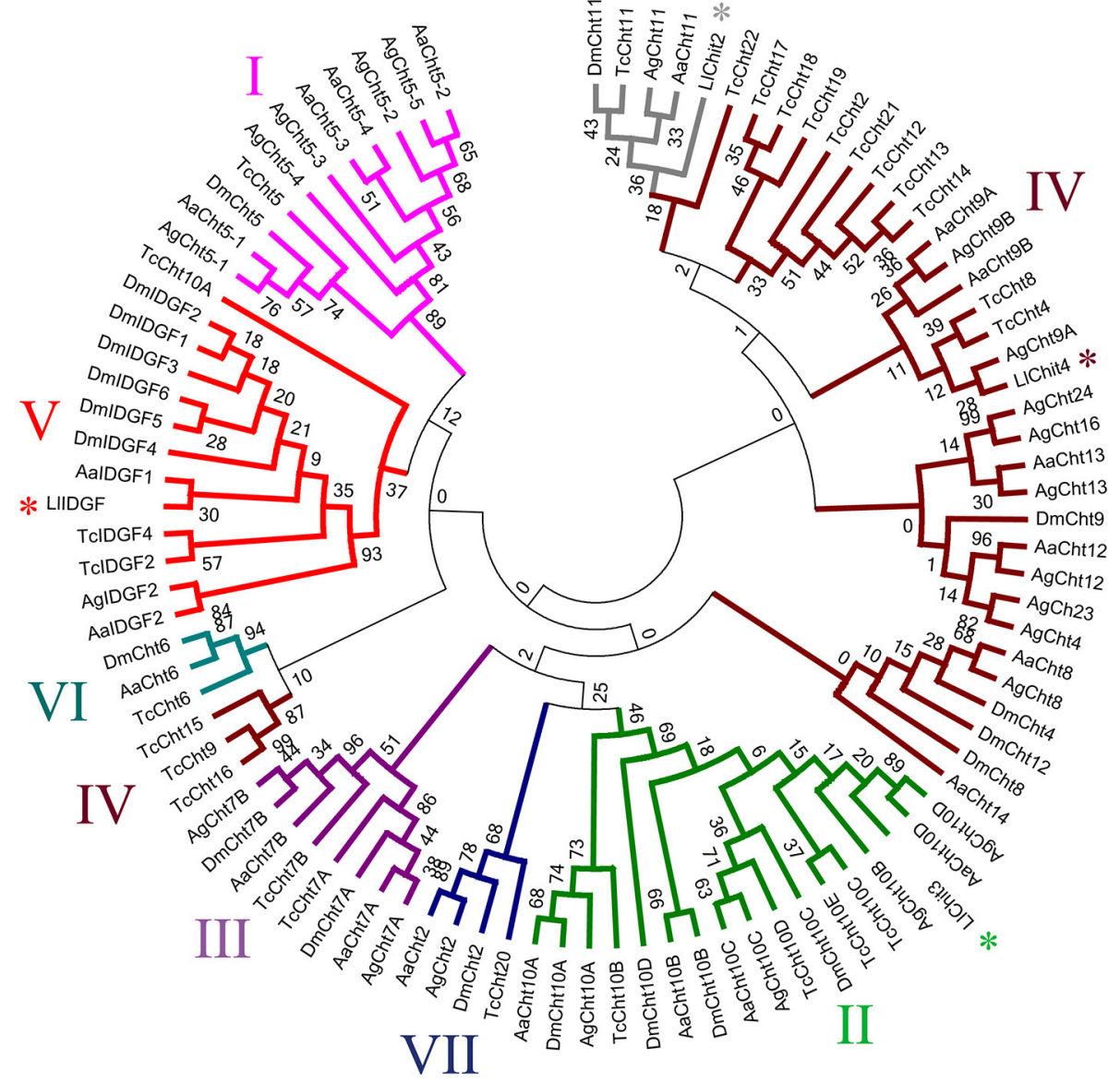

FIGURE 2 | Cladogram of selected insect GH18 protein sequences and positioning of L. longipalpis GH18 (LIChit2, LIChit3, LIChit4, and LIIDGF) in chitinase subgroups. Sequences are from: Aedes aegypti (accession number XP_001657537; XP_001656234; XP_001656233; XP_001656232; XP_001656231; XP_001662588; XP_001650020; XP_001663097; XP_001656054; XP_001655973; XP_001654045; XP_001663568; XP_001655071; XP_001663099; XP_001660745; XP_001660748), Anopheles gambiae (XP_315650; XP_315351; HQ456129; HQ456130; HQ456131; HQ456132; HQ456133; XP_308858; XP_316448; XP_307732; XP_001238192; XP_310662; XP_316142; XP_314312; XP_319801; XP_001688641; XP_316256; XP_001237925), Drosophila melanogaster (NP_477298;
NP_524962; NP_650314; NP 572598; NP_647768; NP_611542; NP_611543; EAA46011; NP_572361; NP_726022; NP_477258; NP_477257; NP_723967; NP_727374; NP_611321; NP_477081) and Tribolium castaneum (NP_001034516; NP_001073567; NP_001034524; XP_967813; NP_001036035; NP_001038094; NP_001038096; NP_001036067; XP_974461; XP_972802; NP_001036034; XP_973005; XP_973077; NP_001034515; XP_972719; XP_973161; XP_973119; XP_970191; NP_001034517; NP_001038095; NP_001038092; NP_001038091. Bootstrap values were obtained by neighbor-joining method (software MEGA 5.05) using 5000 replications. Branches marked with an asterisk correspond to L. longipalpis sequences.
LlGBP2 showed higher expression levels in head and carcass when compared to the gut $(p<0.01)$ (Figure 7C).

LlChit2 and LlChit3 showed no statistically significant differences between any tissue-specific samples (Figures 8A,B, respectively). However, data suggest a slightly higher expression in carcass samples for LlChit3 (Figure 8B).

LlChit 4 was more expressed in the gut tissue when compared to the head and carcasss of sand fly larvae (Figure 8C, $p<0.01$ ). LlChit5 was more expressed in carcass when compared to gut samples $(p<0.01$, Figure $8 D)$. LIIDGF showed significantly higher expression values in carcass when compared to other tissues $(p<0.01$, Figure 8E). LlLysi also showed higher expression levels in carcass compared to the head or intestine $(p<0.01$, Figure 9).

\section{DISCUSSION DIGESTION OF MICROORGANISMS IN DETRITIVORE INSECTS AND SAND FLIES}

Despite the wide variety of dietary sources used by insects in nature, feeding on decaying organic matter (i.e., plant debris and animal feces) is a recurrent evolutionary trait in several insect orders as Dictyoptera, Isoptera, Coleoptera, Diptera. Furthermore, some insects feed on organic matter decomposers such as fungi and are called fungivorous. These groups include some social insects from order Hymenoptera so specialized that they cultivate fungi to feed their own colonies (Chapela et al., 1994). Some species of beetles, such as Dorcus rectus feeds on decaying wood and its associated fungi in nature and can survive under 


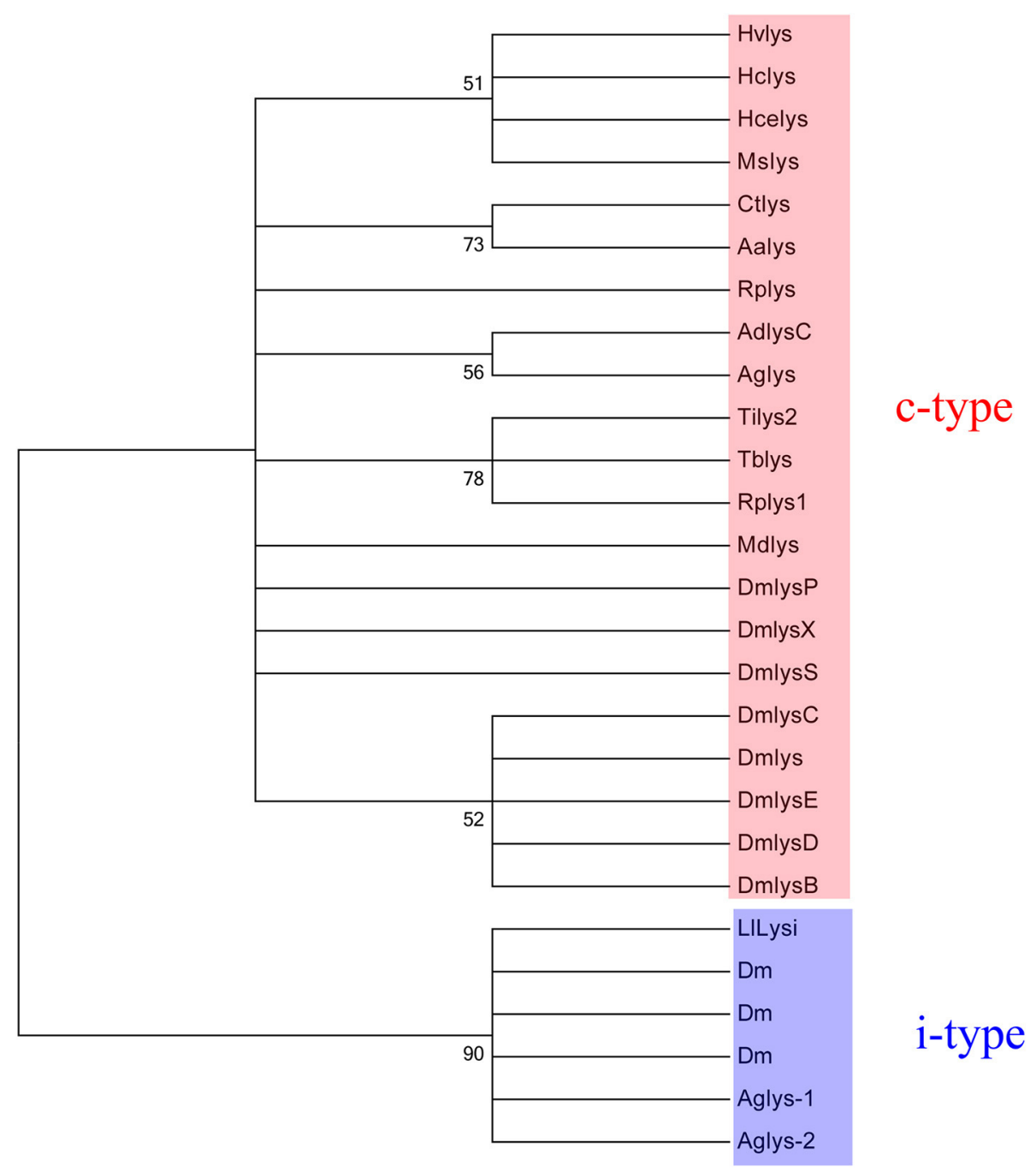

FIGURE 3 | Cladogram of selected insect GH22 protein sequences (c and i-type lysozymes) and LILysi. Sequences are from: Anopheles darlingi (accession number AAB61345), Anopheles gambiae (AAC47326; AY659931; EF492429), Drosophila melanogaster (AAF47445; AAF47453; CAA21317; AAF57939; AAF57940; AAF47452; AAF47451; AAF47450; AAF47449;

AAF47448; CAA80225), Heliothis virescens (AAD00078), Hyalophora cecropia
(AAA29189), Hyphantria cunea (AAA84747), Manduca sexta (AAB31190), Musca domestica (PC4062), Triatoma infestans (AB194387), Triatoma brasiliensis (AAU04569), Rhodnius prolixus (ABX11554; ABX11553), Culex tarsalis (ACJ64375), Aedes aegypti (CAC19819). Bootstrap values were obtained by neighbor-joining method (software MEGA 5.05) using 5000 replications. laboratory conditions fed merely on fungi (Tanahashi et al., 2009).

L. longipalpis is a Dipteran from family Psychodidae and hematofagous females of this species are the main vector of visceral leishmaniasis in the New World. However, little is known about the feeding habits of their larvae in their natural environment. In experimental conditions, larval L. longipalpis and L. intermedia developed better when fed on a diet of fungirich humus which mimics larval substrate in the wilderness (Wermelinger and Zanuncio, 2001). Recently, breeding sites for sand fly larvae were described in the forest located at Amazonas State (Alencar et al., 2011) and in urban and peri urban areas in Southeast Brazil (Casanova et al., 2013). In the forest, sand fly larvae seem to be associated with the humus-rich soil near tree bases, and in urban and peri-urban areas the preferential breeding sites seem to be the soil at chicken sheds. Despite the limitations of these studies, it is quite relevant to perceive the strict association of sand fly larvae with microorganism-rich decaying organic matter in the wild.

Considering that, it is expected that sand fly larvae could exploit microorganisms as bacteria and fungi as a nutritional source. In this respect, their digestive enzymes must be capable of chemically disrupt the cell walls of these microorganisms, in order to release intracellular molecules as nucleic acids, proteins and storage sugars and lipids, which are essential to the insect development and metabolism. The main digestive enzymes related to fungal and bacterial cell disruption in insects are $\beta-1,3$ glucanase, chitinase, and lysozyme. Digesting $\beta$-1,3-glucanases 

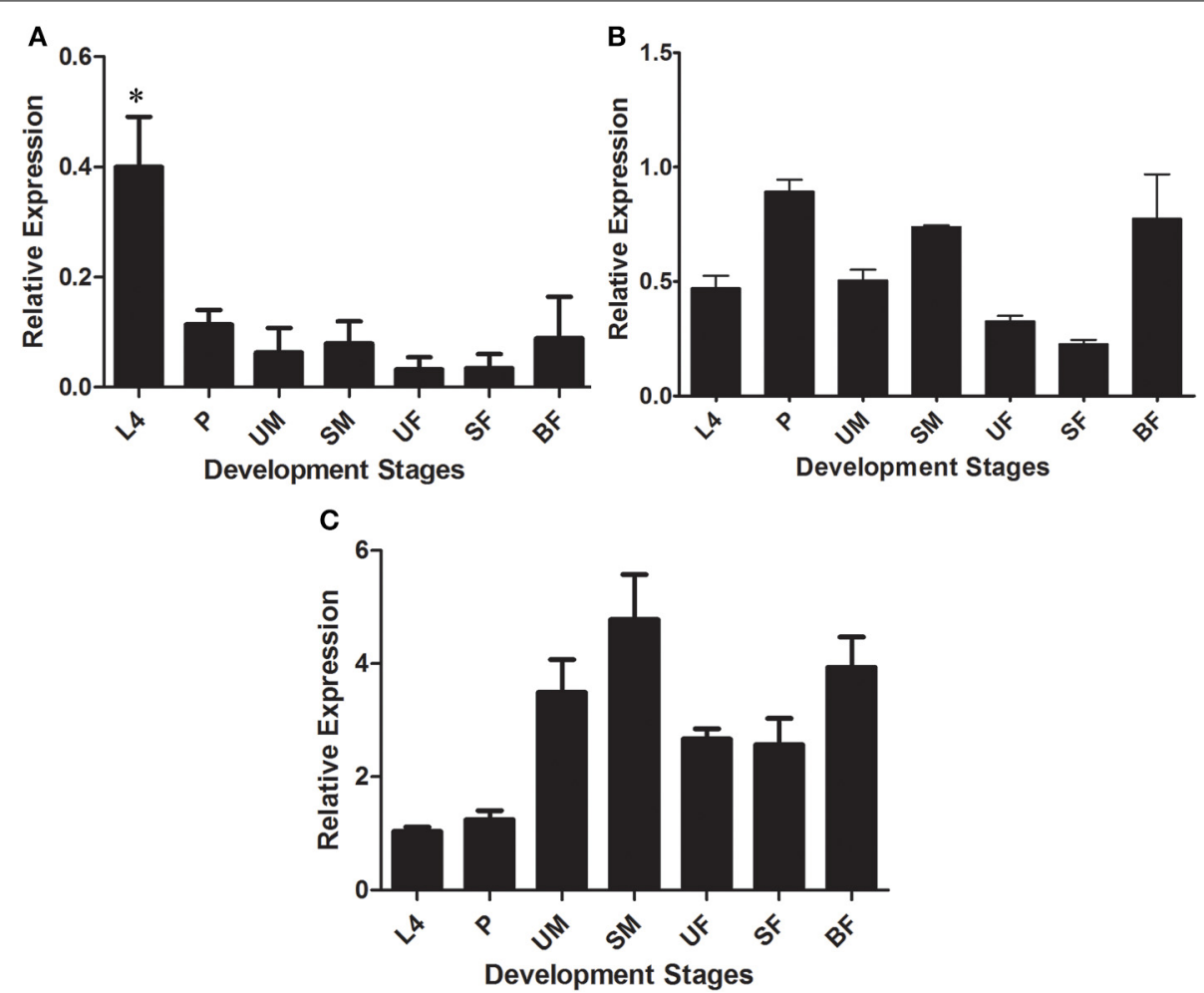

FIGURE 4 | Relative expression of $\beta$-1,3-glucanases (LI $\beta$ Glu) (A) and $\beta$-glucan binding proteins (LIGBP1 and LIGBP2) (B,C, respectively) in different stages of development of Lutzomyia longipalpis as determined by Multiplex RT-PCR (Ribosomal protein $\mathbf{6 0}$ used as constitutive gene). The experimental groups used in the experiments were larvae (L4), pupae
(P), unfed male adults (UM), sugar-fed male adults (SM), unfed female adults (UF), sugar-fed female adults (SF), and blood-fed female adults (BF). Bars represent the mean \pm SE from 3 independent experiments. The statistics were done using ANOVA with Turkey post-test and student's $t$-test. Asterisks indicate statistically significant differences in the samples. from GHF16 were already described in detritivore insects from orders Dictyoptera (Genta et al., 2003), Coleoptera (Genta et al., 2009), Isoptera (Lucena et al., 2011). Digestive chitinases from GHF18 were described in detritivore Coleoptera (Genta et al., 2006), and digestive Lysozymes (GHF22) are a common feature of Dipteran Cyclorrapha (Terra and Ferreira, 2005). All these enzymes were found in a recent report on sand fly larval gut activities (Moraes et al., 2012) but, contrarily to the examples above, sand fly enzymes could not be molecularly identified by traditional biochemical techniques, due to the minimal size of these insects. In spite of that, our bioinfomatic and expression analysis of selected transcripts in L. longipalpis suggest that these larvae use GHF16 and GHF18 in their digestion, as observed in other insect orders.

\section{FUNCTIONAL SPECIALIZATION OF GHF16 MEMBERS IN $L$ LONGIPALPIS}

The in silico search for GH-like sequences in a L. longipalpis EST library showed the presence of three clones highly similar to family GH16 proteins (NSFM-14g04, NSFM-111b04, and NSFM-14b06). Previous sequence comparisons and phylogenetic analysis supported an evolutionary relationship between $\beta$-1,3glucanases and $\beta$-glucan binding proteins from GHF16 (Pauchet et al., 2009; Bragatto et al., 2010; Hughes, 2012). The most evident features which distinguish these two functional groups are
(1) the presence of catalytic glutamates in a conserved region which correspond to the active site in $\beta$-1,3-glucanases and (2) the presence of a conserved 100 amino acid $\mathrm{N}$-terminal extension in $\beta$-glucan binding proteins. It has been proposed that the animal $\beta$-1,3-glucanase ancestral gene suffered a duplication before the differentiation of arthropods and molluscs (Bragatto et al., 2010) and, in this respect, insects should bear at least two copies of genes from GHF16.

Pauchet et al. (2009) also divided clades of Lepidopteran GHF16 sequences according to the absences or presences of catalytic residues. The authors suggested that such division happened through a duplication event of a gene in a common ancestor. This originated two major groups with different functions in insects: digestion and immune signaling. Recently, Hughes (2012) showed that GHF16 Pathogen Recognition Receptors (PRRs, which includes $\beta$-1,3-glucan binding proteins) and $\beta-1,3$ glucanase sequences are present in orders Coleoptera, Diptera and Lepidoptera, and suggested that this gene duplication event occurred before divergence of these holometabolous orders. The author also exhibited that only PRR-like sequences are found within Exopterigota (hemimetabolous), while only glucanaselike sequences are found in Isoptera. Such evidence supports the hypothesis that these two subfamilies underwent events of gene duplication before the origin of holometabolous insects (i.e., during the Carboniferous, approximately 300 million years ago). 

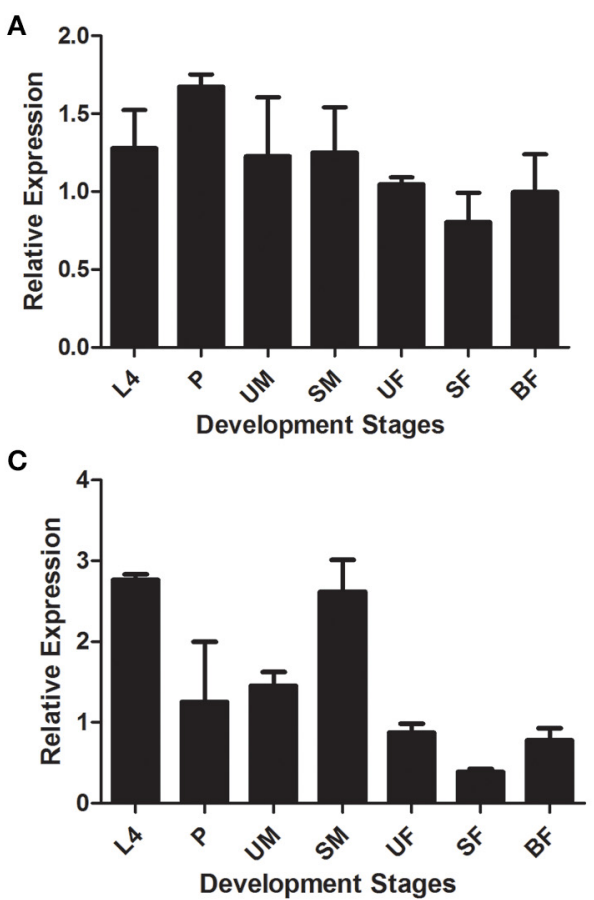

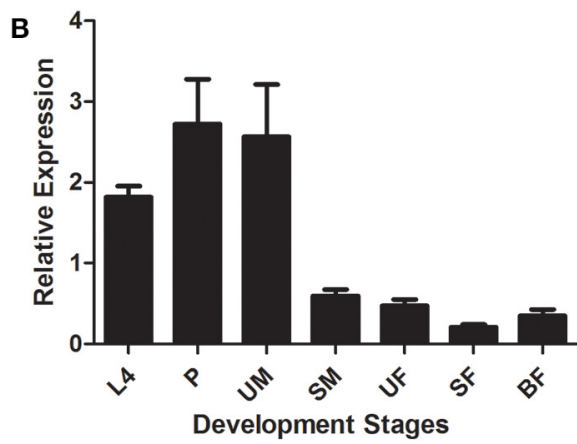

D

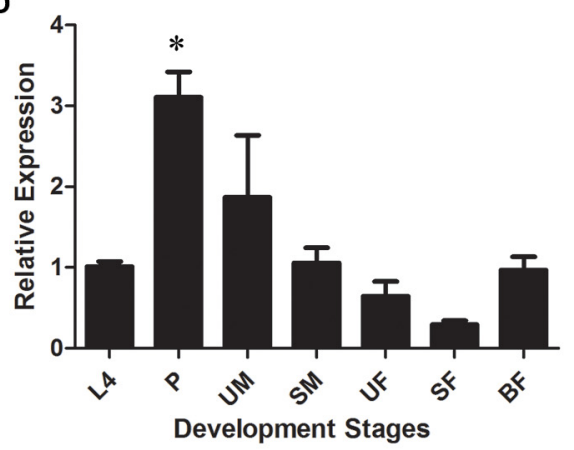

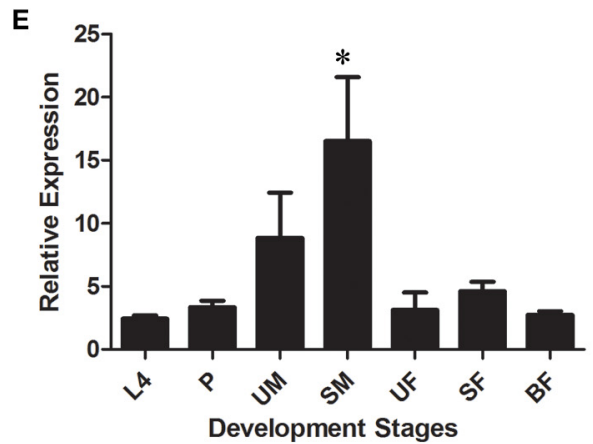

FIGURE 5 | Relative expression of GHF18 members in different stages of development of Lutzomyia longipalpis as determined by Multiplex RT-PCR (Ribosomal protein $\mathbf{6 0}$ used as constitutive gene). Chitinases amplified were: (A) LIChit2; (B) LIChit3; (C) LIChit4; (D) LIChit5, and (E) LIIDFG. The experimental groups used in the experiments were larvae (L4), pupae (P), unfed male adults (UM), sugar-fed male adults (SM), unfed female adults (UF), sugar-fed female adults (SF), and blood-fed female adults (BF). Bars represent the mean \pm SE from 3 independent experiments. The statistics were done using ANOVA with Turkey post-test and student's $t$-test. Asterisks indicate statistically significant differences in the samples.
Based on the criteria described above, NSFM-14g04 was classified as a $\beta$-1,3-glucanase (Ll $\beta$ Glu) while NSFM-111b04 and NSFM-14b06 were assigned to the $\beta$-glucan binding protein group (as LlBGP1 and LlBGP2). Alignment of these sequences with other insect GH16 showed that clone NSFM-14g04 contains the two glutamate residues which are important for catalysis. In contrast, NSFM-111b04 and NSFM-14b06 lack these residues, which suggests that these putative proteins do not have enzymatic activity. The presence of the $\mathrm{N}$-terminal region typical for $\beta$-glucan binding proteins was not confirmed in LlGBP1, because this sequence is truncated at the $\mathrm{N}$-terminal, but was confirmed in LlGBP2. Ll $\beta$ Glu, as expected, does not contain this extension, which was confirmed as Ll $\beta$ Glu appears as a complete coding sequence with a putative signal peptide. The presence of a putative signal peptide in Ll $\beta$ Glu and LlGBP2 sequences is coherent with the observations that some digestive insect $\beta$-1,3-glucanases follow the exocytic route for secretion (Bragatto et al., 2010) and that $\beta$-glucan binding proteins are soluble proteins secreted to the hemolymph, where they interact with members of the prophenoloxidase activating cascade (Lee et al., 2004).

The expression pattern of GHF16 members in L. longipalpis corroborated the classification based on sequence features. Ll $\beta \mathrm{Glu}$ was more expressed in larvae and, at this stage, its expression is gut-specific. Gut-specific $\beta$-1,3-glucanases from GHF16 were already described in Lepidoptera (Pauchet et al., 2009; Bragatto et al., 2010), Coleoptera (Genta et al., 2009), and Isoptera (Bulmer et al., 2009). To our knowledge, this is the first description of this kind of protein sequences in Diptera. The role of insect gut $\beta$-1,3-glucanases is still controversial, as they were implicated in digestion of fungi and plant cell wall polysaccharides in 
some insects (Dictyoptera, Genta et al., 2003; Orthoptera, Genta et al., 2007, Coleoptera, Genta et al., 2009) but in recognition of gut-pathogens in others (Lepidoptera, Pauchet et al., 2009; Isoptera, Bulmer et al., 2009). Considering the detritivore habit

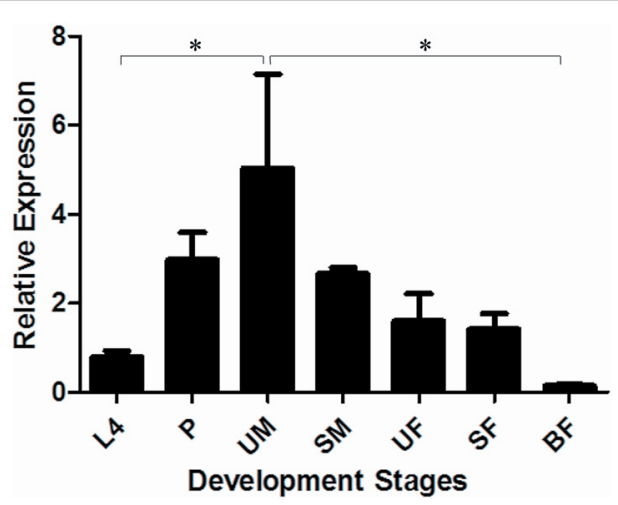

FIGURE 6 | Relative expression of lysozyme (LILysi) in different stages of development of Lutzomyia longipalpis as determined by Multiplex RT-PCR (Ribosomal protein $\mathbf{6 0}$ used as constitutive gene). The experimental groups used in the experiments were larvae (L4), pupae (P), unfed male adults (UM), sugar-fed male adults (SM), unfed female adults (UF), sugar-fed female adults (SF), and blood-fed female adults (BF). Bars represent the mean \pm SE from 3 independent experiments. The statistics were done using ANOVA with Turkey post-test and student's $t$-test. Asterisks indicate statistically significant differences in the samples. of L. longipalpis larvae in the laboratories, with the ingestion of significant amounts of fungal tissue in the food (Moraes et al., 2012), we are tempted to suppose that the main role of LlBGlu is the disruption of ingested fungal cells, but more functional studies are necessary to confirm this hypothesis.

At the same time, LlGBP1 and LlGBP2 showed low levels of expression in the larvae and, at this stage, low levels of expression in the gut. In this respect, a role for these genes in larval digestion can be ruled out. Besides that, the expression patterns of LlGBP1 and LlBGP2 strongly suggest that they are involved in defense against pathogens, as they are expressed in tissues and stages more susceptible to infection. Both genes are highly expressed in males fed with sugar and blood fed females. The ingestion of bacterial pathogens by sand flies in sugar meals is a current topic of investigation (Telleria et al., 2013), as well as the multiplication of these micro organisms inside the blood meal in the sand fly gut (DiazAlbiter et al., 2012). The fact that sand flies mount strong defenses in the gut during these physiological conditions suggests that they are fighting pathogens and, in fact, interfere with this phenomena result in activation of the prophenoloxidase cascade and insect death (Diaz-Albiter et al., 2011).

Nevertheless, there are subtle differences in the expression patterns between LIGBP1 and LlGBP2. LlGBP1 is highly expressed during the pupal stage, as well as LlGBP2 is highly expressed in unfed males. In this respect, these genes behave like their putative homologs in Drosophila, where GNBP1 (CG6895-PA), GNBP2 (CG4144), and GNBP3 (CG5008) are not expressed in
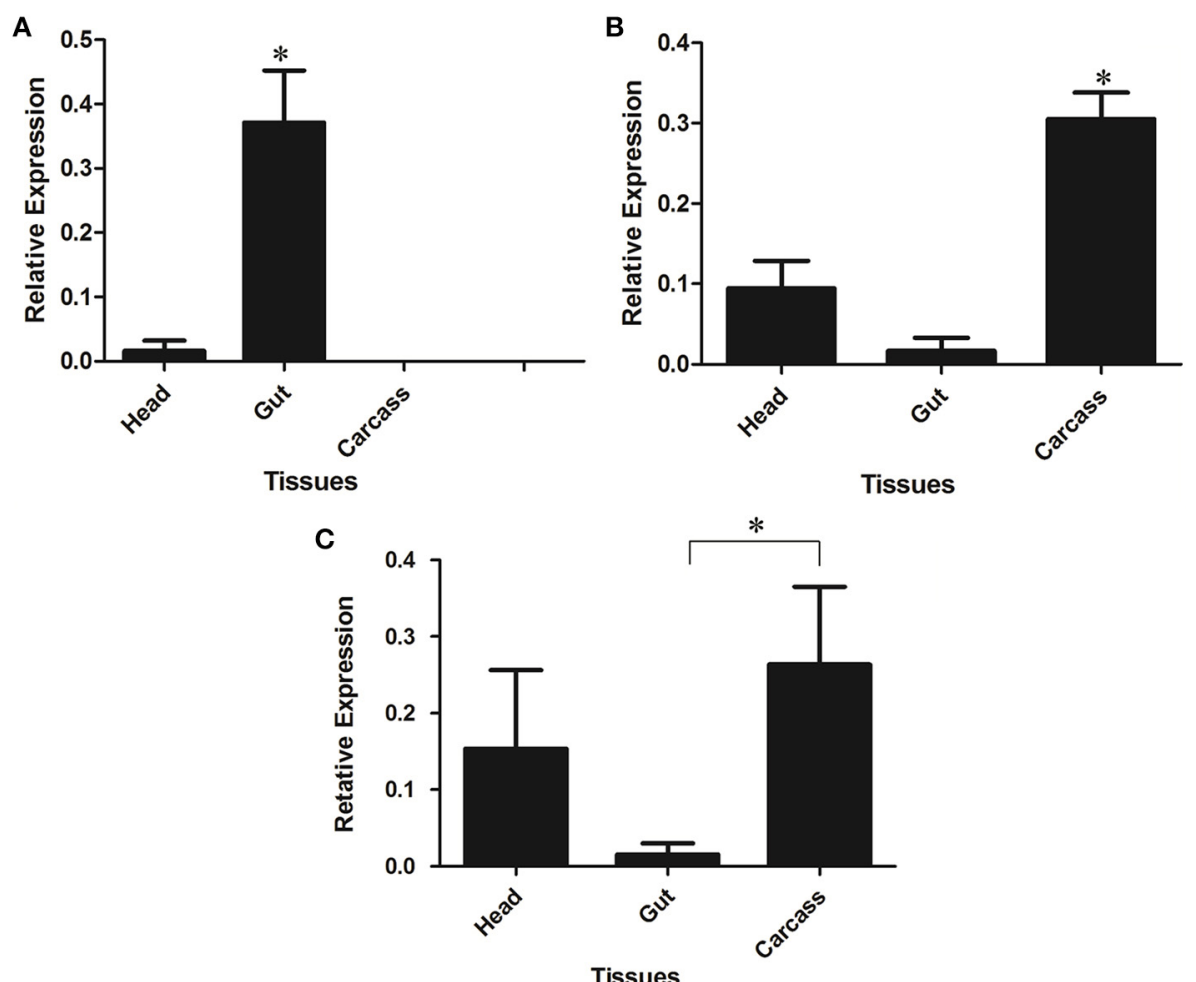

Tissues

Tissues

FIGURE 7 | Relative expression of GHF16 (A) $\beta$-1,3-glucanase (LI $\beta$ Glu) and (B) $\beta$-glucan binding proteins LIGBP1 and (C) LIGBP2 in different tissues of Lutzomyia longipalpis L4 larvae as determined by Multiplex RT-PCR (Ribosomal protein 60 used as constitutive gene). Bars represent the mean \pm SE from 5 independent experiments. The statistics were done using ANOVA with Turkey post-test and student's $t$-test. Asterisks indicate statistically significant differences in the samples. 

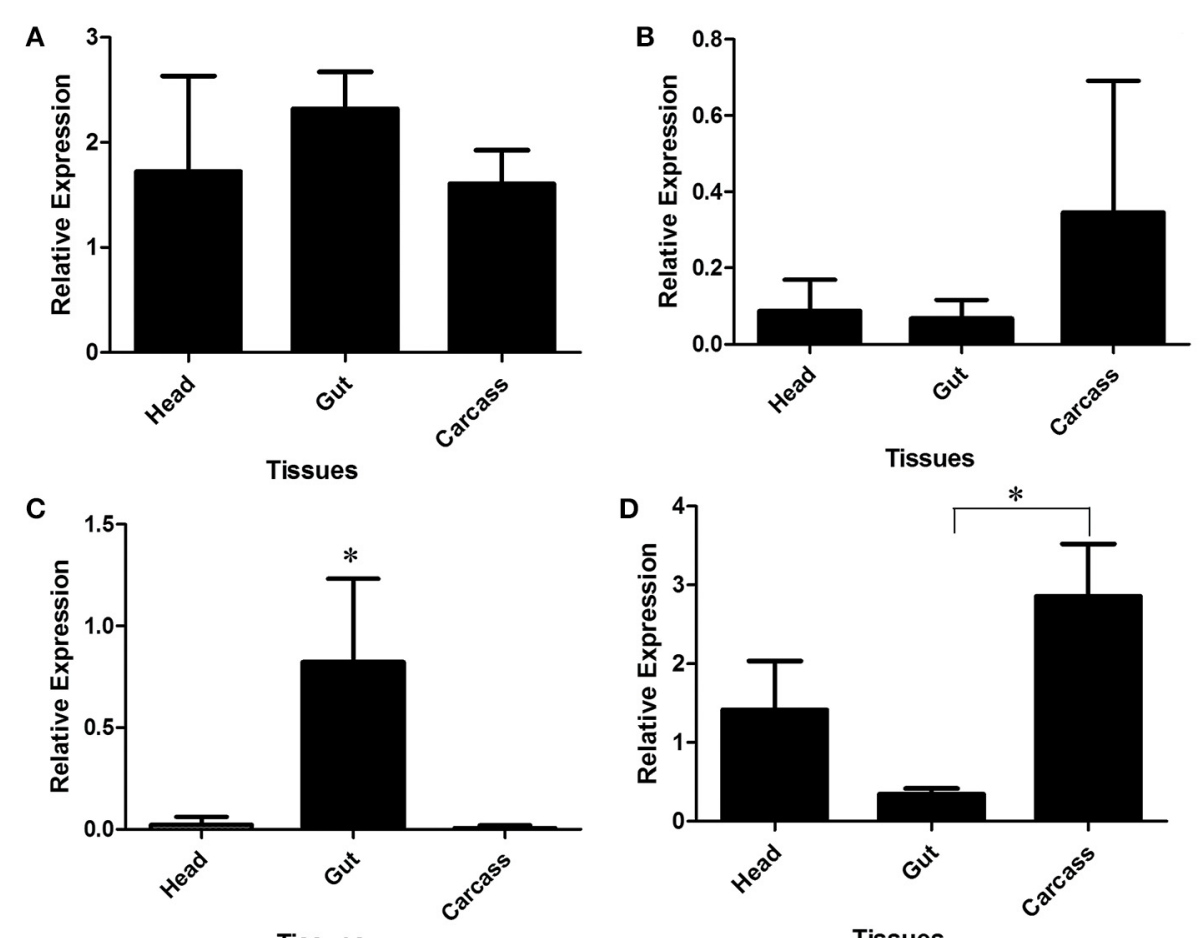

Tissues

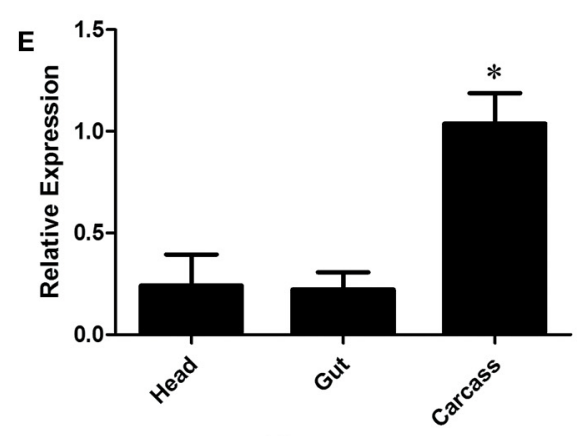

Tissues

FIGURE 8 | Relative expression of GHF18 chitinases and chitinase-like proteins in different tissues of Lutzomyia longipalpis L4 larvae as determined by Multiplex RT-PCR (Ribosomal protein $\mathbf{6 0}$ used as constitutive gene). Chitinases amplified were: (A) LIChit2; (B) LIChit3; (C)
LIChit4; (D) LIChit5 and (E) LIIDGF. Bars represent the mean \pm SE from 5 independent experiments. The statistics were done using ANOVA with Turkey post-test and student's $t$-test. Asterisks indicate statistically significant differences in the samples. the midgut at any stage, with higher expression levels in the embryo (GNBP2 and 3), pupae (all GNBPs) and adults (GNBP2 and 3), and preferential expression in the carcass or head (St. Pierre et al., 2014). It is possible that LlGBP1 is related to the prevention of infections during the pupal stage, which is extremely sensitive to infections. Besides that, it has been shown that unfed sand flies carry a significant amount of bacteria from the larval stage (Sant'Anna et al., 2014), which suggests that LlGBP2 could be involved in the specific protection against these recalcitrant microorganisms. The trans stadial passage of bacteria is a well-documented phenomenon in sand flies (Volf et al., 2002), and may have important implications in the development of strategies for the blocking of vectorial transmission of pathogens based in paratransgenesis. However, more functional studies are needed to confirm the roles of LlGBP1 and LlGBP2, especially after challenge with insect pathogens.

\section{FUNCTIONAL SPECIALIZATION OF GHF18 MEMBERS IN $L$. LONGIPALPIS}

Five sequences from GHF18 were retrieved from the L. longipalpis EST library (NSFM-96h07, NSFM-154b12, NSFM-88d12, NSFM-24g06, and NSFM-18f06). According to amino acid similarities, domain compositions, and phylogenetic analysis, insect proteins from GHF18 are classified into 8 groups, I-VIII (Zhang et al., 2011). A key feature in these proteins is the presence of a catalytic glutamate in the sequence DWEYP at the consensus region 2 (CR2), which is used as a marker for enzymatic activity. GHF18 proteins without this residue are named chitinase-like 


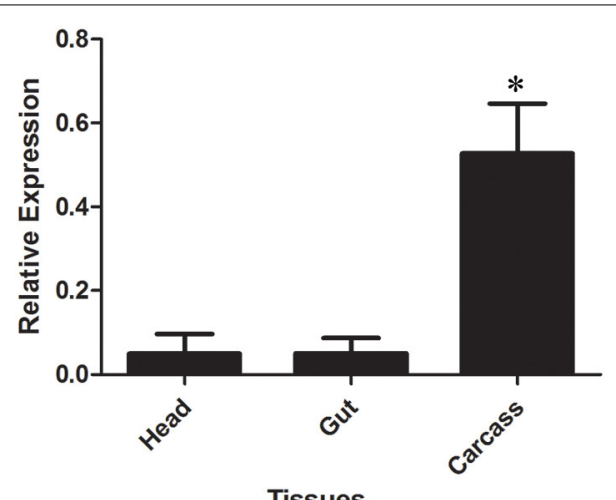

Tissues

FIGURE 9 | Relative expression of i-type lysozyme (LILysi) in different tissues of Lutzomyia longipalpis L4 larvae as determined by Multiplex RT-PCR (Ribosomal protein $\mathbf{6 0}$ used as constitutive gene). Bars represent the mean \pm SE from 5 independent experiments. The statistics were done using ANOVA with Turkey post-test and student's $t$-test. Asterisks indicate statistically significant differences in the samples.

proteins and considered devoid of hydrolytic activity. Besides that, functional studies were able to incriminate groups I and II of insect chitinases in chitin hydrolysis during molting, group III in the distension of wings and abdomen during morphogenetic development, and group IV as digestive enzymes. Group $\mathrm{V}$ is devoid of catalytic activity and is referred as Imaginal Disk Growth Factors, promoting cell proliferation in imaginal disks. Groups VI-VIII were described based only in sequence attributes, with no clear functional role assessed to this moment. In general, chitinases from all groups but IV are expressed in all stages of development, while the expression of group IV chitinases is restricted to specific stages. Many group IV chitinases are larvalspecific, and gut-specific genes, being considered primarily as digestive enzymes. Some are involved in digestion or turnover of the peritrophic membrane (Ramalho-Ortigão and Traub-Csekö, 2003), but others, which lack the characteristic C-terminal Chitin Binding Domain, are believed to act in disruption of fungal cell walls without affecting the PM structure (Genta et al., 2006).

The domain organization of all L. longipalpis GHF18 sequences present in the ESTs database could not be assessed, because only two of them (NSFM-154b12 and NSFM-18f06) code for complete ORFs. These complete sequences contain a putative $\mathrm{N}$-terminal signal sequence, which is a common feature in GHF18 insect chitinases (Zhang et al., 2011). This is coherent with chitinase putative secretion to the molting fluid, intestinal lumen, or to the extracellular matrix where it exerts its action on imaginal disk cells (Arakane and Muthukrishnan, 2010).

In spite of lacking complete $\mathrm{N}$ - or C-terminal ends, all GHF18 proteins from $L$. longipalpis were assigned to functional groups of insect chitinases II, IV, V, or VIII, being named LlChit2 (group VIII), 3 (group II), 4 and 5 (Group IV) and LlIDGF (group V). From these sequences, only LlChit2 and LlChit 3 contain the conserved catalytic glutamate at CR2, being probably true chitinases. LIIDGF is a chitinase-like protein, and it was not possible to check for the presence of the catalytic glutamate in the case of LlChit4, because CR2 was missing in this sequence. In spite of that, LlChit4 is probably an active enzyme, as it groups with other true chitinases from group IV and contain the conserved PFAM domain Glyco_hydro_18 (PF00704). LlChit5 also aligned with proteins of group IV, but was not included in the phylogenetic analysis because the PFAM domain PF00704 was missing in its sequence. Because of that, the presence of the catalytic glutamate in region CR2 could not be really assessed, as its apparent substitution in LlChit5 sequence could be the result of the forced alignment of its truncated sequence. Another possibility is that LlChit5 use an alternative basic hydrolysis mechanism, based only in the nucleophilic catalysis from the acetamido group of the substrate (Hashimoto et al., 2000). More evidence is necessary to ascertain the catalytic nature of LlChit4 and LlChit5.

Chitinases from subgroups II, V, and VIII are typically expressed in all stages of insect development (Zhang et al., 2011). The expression pattern observed for LlChit2, 3, 5, and LlIDGF is consistent with this behavior. Indeed, LlChit2, 3, and 5 reach their highest expression levels in the pupal stage, and LIIDGF is more expressed in adult males. Besides that, in the larvae these genes are more expressed in the carcass (LlChit3, 5, and LlIDGF) or equally expressed in all larval tissues (LlChit2). These data strongly suggest that LlChit2, 3, 5, and LlIDGF are not involved in the digestion of sand fly larvae.

Interestingly, LlChit4, which is related to typical insect digestive chitinases from group IV, is more expressed in larvae and sugar fed males. Type IV chitinases contain a signal peptide, a single catalytic domain and mostly no CBDs (Genta et al., 2006). Additionally, LlChit4 is more expressed in the gut of larvae, which strongly suggest that this transcript correspond to the larval digestive chitinase of L. longipalpis. The higher expression in sugar fed males could be related to the proliferation of microorganisms in the gut of these insects, but this hypothesis still needs confirmation.

Similar results were observed by Khajuria et al. (2010), who demonstrated by phylogenetic analysis that a chitinase (OnCht) predominantly expressed in the gut of larval Ostrinia nubilalis was a type IV chitinase. A digestive chitinase was also found in the midgut of T. molitor larvae (Genta et al., 2006, TmChi).

In several hematophagous insects, digestive chitinases have an extremely important role in the degradation of type I peritrophic matrix (PM I). Zhang et al. (2011) showed expression of numerous chitinases in Anopheles gambiae, among them, a chitinase mainly expressed in the gut of adults (AgCht8).

Previous studies made by Ramalho-Ortigão and Traub-Csekö (2003) demonstrated the expression of chitinase (LlChi1) in the midgut of adult female L. longipalpis, whose expression seems to be induced after blood feeding. However, so far the expression of chitinases in larvae of $L$. longipalpis has not been demonstrated and studies to pursue a chitinase important for digestion of microorganisms present in the diet of the larvae are still scarce. The data obtained suggest that LlChit4 probably has that role.

To our knowledge, this is the first description of a larval gut chitinase sequence in sand flies. Larval gut chitinase activities were already described in L. longipalpis (Moraes et al., 2012) and Aedes aegypti (Souza-Neto et al., 2003), these enzymes being probably involved in the digestion of chitin rich structures as the fungal cell wall. Gut-specific expression of chitinase genes in 
Diptera was already described in Anopheles gambiae larvae (Zhang et al., 2011), where group IV chitinases AgCht8 and AgCht13 are majorly expressed only in the midgut at this stage. It is interesting to notice that in some cases dipteran larval digestive chitinases seem to be coded by genes different from their adult counterparts, a pattern already observed for other gut genes, as trypsins and peritrophins (Venancio et al., 2009).

\section{ANALYSIS OF THE GHF22 SEOUENCE FOUND IN ADULT ESTS LIBRARY OF LUTZOMYIA LONGIPALPIS}

Lysozymes are present in a wide variety of organisms (from viruses to plants and animals) and can be classified based on sequence similarity into 5 groups: type c, type g, type i, plant, and bacterial lysozymes (Bachali et al., 2002). Insect lysozymes are commonly members of family $\mathrm{GH} 22$, being involved in the arthropod innate immune response against bacteria but also in the digestion of food microorganisms in detritivores (Jollès and Jollès, 1984; Hultmark, 1996). The most well characterized insect digestive lysozyme is the gut activity from Musca domestica larvae. This enzyme is a c-type lysozyme (Cançado et al., 2007) with specializations in its structure which are related to its optimum $\mathrm{pH}$ at the midgut strong acidic conditions (Cançado et al., 2010), which is a typical feature of Diptera Cyclorrapha (Terra and Ferreira, 1994). As the midgut luminal $\mathrm{pH}$ of sand fly larvae is strongly alkaline (Do Vale et al., 2007) it could be possible that their digestion of bacteria rely on a different type of lysozyme.

In recent years studies have revealed the existence in insects of a second family of lysozymes, type $i$, for which at least 20 examples have been identified in the phylum Mollusca, Nematoda, Annelida, and Arthropoda (Ito et al., 1999; Zavalova et al., 2000; Bachali et al., 2002; Paskewitz et al., 2008).

Therefore, considering the possible role of lysozyme in the degradation of dietary microorganisms in sand flies, a search was also made for GHF22 members in the EST database of L. longipalpis. Our analysis revealed only one transcript similar to insect lysozymes, called LlLysi (NSFM-123b01), whose sequence was complete including a putative $\mathrm{N}$-terminal signal peptide. Moreover, comparison of this sequence in the Pfam database showed that LlLysi contains a destabilase domain.

Similarity to Destabilase is common among i-type lysozymes. The leech destabilase has such a name from their ability to break or destabilize fibrin blood clots in the ingested blood (Zavalova et al., 2000). Using this relationship, the leech destabilase was used as a pattern to pursue i-lysozymes in the genome of Anopheles gambiae (Paskewitz et al., 2008).

Aiming to classify the lysozyme found in the EST library of L. longipalpis, we performed a phylogenetic analysis using the neighbor-joining algorithm. In this analysis it was possible to demonstrate the separation of c- or i-lysozymes in two monophyletic groups. LlLysi was classified in this analysis as ilysozyme. To our knowledge, this is the first description of this type of lysozyme in sand flies.

LlLysi was expressed throughout all developmental stages of L. longipalpis, expression being the highest in unfed males. This could be related to the presence of high amounts of bacteria in recently emerged adults of $L$. longipalpis (Sant'Anna et al., 2014).
Besides that, a very low level of LlLysi expression was found in L4 larvae, being concentrated in the carcass, excluding the possibility of LlLysi being a larval digestive enzyme. Similar studies were done in the mosquito A. gambiae, which produces two itype lysozymes, called Lys i-1 and i-2. Lys i-1 was expressed in all stages of mosquito development, being more expressed in the ovaries of females, followed by Malpighian tubules and fat body. Lys i-2 was more abundant in the fat body of adults (Paskewitz et al., 2008).

Thus, in sand flies, the lysozyme gene involved in the larval digestion of bacteria, which corresponds to the activity described by Moraes et al. (2012) is different from the i-lysozyme transcripts represented in adult EST databases. Our studies raise the interesting possibility that sand fly larval gut lysozyme could be in fact more related to the c-lysozymes from other Diptera, but more studies are necessary aiming the molecular identification of this enzyme.

\section{CONCLUSION}

The comparative analysis of sequences present in EST databases as well as the determination of expression patterns during development and the tissue location of transcripts from families GH16 and GH18, allowed us to identify the genes related to the $\beta$ 1,3-glucanase and chitinase which are involved in digestion of microorganisms in larvae of L. longipalpis. However, the sequence of lysozyme present in adult EST databases (LlLys, family GH22) is apparently a type i Lysozyme involved in immunity, having no relationship to larval digestion of bacteria. To our knowledge, this is the first molecular identification of digestive enzymes in the larvae of sand flies, which are important vectors of several diseases, including Leishmaniasis. These findings may have a profound impact on the understanding of the biology of these disease vectors, as well as for the development of new strategies for vector control.

\section{AUTHOR CONTRIBUTIONS}

Conception and design of the work: Rod J. Dillon, Maurício R. V. Sant'Anna, Fernando A. Genta. Obtainment of experimental data: Caroline da Silva Moraes, Hector M. Diaz-Albiter, Maiara do Valle Faria, Maurício R. V. Sant'Anna, Fernando A. Genta. Data analysis: Caroline da Silva Moraes, Maiara do Valle Faria, Hector M. Diaz-Albiter. Writing and revision of the manuscript: Caroline da Silva Moraes, Hector M. Diaz-Albiter, Rod J. Dillon, Fernando A. Genta.

\section{ACKNOWLEDGMENTS}

This research was supported by the Research Agencies FAPERJ, CNPq, CAPES and FIOCRUZ (Brazil), The LeverHulme Trust (UK) and CONACYT (Mexico). Caroline da Silva Moraes received a Ph.D grant from CAPES (Cellular and Molecular Biology Postgraduation Program) and MVF undergraduate funding from FAPERJ and CNPq/PIBIC. Hector M. DiazAlbiter is a postdoctoral fellow from the CNPq/CAPES Science Without Borders Program. Maurício R. V. Sant'Anna, Rod J. Dillon and Fernando A. Genta are staff members of their respective departments. The authors thank Reginaldo Peçanha Brazil, Eloi de Souza Garcia and Patricia de Azambuja Penna 
(IOC-FIOCRUZ) for fruitful discussions and Tainá Neves Ferreira (IOC-FIOCRUZ) and Davina Moor (LSTM) for technical assistance.

\section{SUPPLEMENTARY MATERIAL}

The Supplementary Material for this article can be found online at: http://www.frontiersin.org/journal/10.3389/fphys.2014.

\section{6/abstract}

\section{REFERENCES}

Alencar, R. B., de Queiroz, R. G., and Barrett, T. V. (2011). Breeding sites of phlebotomine sand flies (Diptera: Psychodidae) and efficiency of extraction techniques for immature stages in terra-firme forest in Amazonas State, Brazil. Acta Tropica 118, 204-208. doi: 10.1016/j.actatropica.2010.10.012

Amaro, F., Luz, T., Parreira, P., Marchi, A., Ciufolini, M. G., and Alves, M. J. (2012). Serological evidence of Toscana virus infection in Portuguese patients. Epidemiol. Infect. 140, 1147-1150. doi: 10.1017/S0950268811001403

Arakane, Y., and Muthukrishnan, S. (2010). Insect chitinase and chitinase-like proteins. Cell. Mol. Life Sci. 67, 201-216. doi: 10.1007/s00018-009-0161-9

Bachali, S., Jager, M., Hassanin, A., Schoentgen, F., Jollès, P., Fiala-Medioni, A., et al. (2002). Phylogenetic analysis of invertebrate lysozymes and the evolution of lysozyme function. J. Mol. Evol. 54, 652-664. doi: 10.1007/s00239-0010061-6

Bacic, A., Harris, P. J., and Stone, B. A. (1998). "Structure and function of plant cell walls," in The Biochemistry of Plants, eds P. K. Stumpf and E. E. Conn (New York, NY: Academic Press), 297-371.

Bartnicki-Garcia, S. (1968). Cell wall chemistry, morphogenesis, and taxonomy of fungi. Annu. Rev. Microbiol. 22, 87-108. doi: 10.1146/annurev.mi.22.100168. 000511

Bragatto, I., Genta, F. A., Ribeiro, A. F., Terra, W. R., and Ferreira, C. (2010). Characterization of a $\beta$-1,3-glucanase active in the alkaline midgut of Spodoptera frugiperda larvae and its relation to $\beta$-glucan-binding proteins. Insect Biochem. Mol. Biol. 40, 861-872. doi: 10.1016/j.ibmb.2010.08.006

Brazil, R. P., and Brazil, B. G. (2003). "Binomia," in Flebotomíneos do Brasil, ed E. F. Rangel and R. Lainson (Rio de Janeiro: Editora Fiocruz), 257-274.

Bulmer, M. S., Bachelet, I., Raman, R., Rosengaus, R. B., and Sasisekharan, R. (2009). Targeting an antimicrobial effector function in insect immunity as a pest control strategy. Proc. Natl. Acad. Sci. U.S.A. 106, 12652-12657. doi: 10.1073/pnas.0904063106

Cançado, F. C., Barbosa, J. A., and Marana, S. R. (2010). Role of the triad N46, S106 and T107 and the surface charges in the determination of the acidic $\mathrm{pH}$ optimum of digestive lysozymes from Musca domestica. Comp. Biochem. Physiol. B Biochem. Mol. Biol. 155, 387-395. doi: 10.1016/j.cbpb.2010.01.001

Cançado, F. C., Valério, A. A., Marana, S. R., and Barbosa, J. A. (2007). The crystal structure of a lysozyme c from housefly Musca domestica, the first structure of a digestive lysozyme. J. Struct. Biol. 160, 83-92. doi: 10.1016/j.jsb.2007.07.008

Casanova, C., Andrighetti, M. T., Sampaio, S. M., Marcoris, M. L., Colla-Jacques, F. E., and Prado, A. P. (2013). Larval breeding sites of Lutzomyia longipalpis (Diptera: Psychodidae) in visceral leishmaniasis endemic urban areas in southeastern Brazil. PLoS Negl. Trop. Dis. 7:e2443. doi: 10.1371/journal.pntd. 0002443

Chapela, I. H., Rehner, S. A., Schultz, T. R., and Mueller, U. G. (1994). Evolutionary history of the symbiosis between fungus-growing ants and their fungi. Science 266, 1691-1694. doi: 10.1126/science.266.5191.1691

Coutinho-Abreu, I. V., Sharma, N. K., Robles-Murguia, M., and Ramalho-Ortigao, M. (2010). Targeting the midgut secreted PpChitl reduces Leishmania major development in its natural vector, the sand fly Phlebotomus papatasi. PLos Negl. Trop. Dis. 4:e901. doi: 10.1371/journal.pntd.0000901

De la Vega, H., Specht, C. A., Liu, Y., and Robbins, P. W. (1998). Chitinases are a multi-gene family in Aedes, Anopheles and Drosophila. Insect Mol. Biol. 7, 233-239.

Diaz-Albiter, H., Mitford, R., Genta, F. A., Sant'Anna, M. R., and Dillon, R. J. (2011). Reactive oxygen species scavenging by catalase is important for female Lutzomyia longipalpis fecundity and mortality. PLoS ONE 6:e17486. doi: 10.1371/journal.pone.0017486

Diaz-Albiter, H., Sant'Anna, M. R., Genta, F. A., and Dillon, R. J. (2012). Reactive oxygen species-mediated immunity against Leishmania mexicana and Serratia marcescens in the sand phlebotomine fly Lutzomyia longipalpis. J. Biol. Chem. 287, 23995-24003. doi: 10.1074/jbc.M112.376095

Dillon, R. J., and El Kordy, E. (1997). Carbohydrate digestion in sandflies: $\alpha-$ glucosidase activity in the midgut of Phlebotomus langeroni. Comp. Biochem. Physiol. 116B, 35-40. doi: 10.1016/S0305-0491(96)00195-2

Dillon, R. J., Ivens, A. C., Churcher, C., Holroyd, N., Quail, M. A., Rogers, M. E., et al. (2006). Analysis of ESTs from Lutzomyia longipalpis sand flies and their contribution toward understanding the insect-parasite relationship. Genomics 88, 831-840. doi: 10.1016/j.ygeno.2006.06.011

Do Vale, V. F., Pereira, M. H., and Gontijo, N. F. (2007). Midgut pH profile and protein digestion in the larvae of Lutzomyia longipalpis. J. Insect Physiol. 53, 1151-1159. doi: 10.1016/j.jinsphys.2007.06.005

Feliciangeli, M. D. (2004). Natural breeding places of phlebotomine sandflies. Med. Vet. Entomol. 18, 71-80. doi: 10.1111/j.0269-283X.2004.0487.x

Genta, F. A., Blanes, L., Cristofoletti, P. T., Do Lago, C. L., Terra, W. R., and Ferreira, C. (2006). Purification, characterization and molecular cloning of the major chitinase from Tenebrio molitor larval midgut. Insect Biochem. Mol. Biol. 36, 789-800. doi: 10.1016/j.ibmb.2006.07.007

Genta, F. A., Bragatto, I., Terra, W. R., and Ferreira, C. (2009). Purification, characterization and sequencing of the major beta-1,3-glucanase from the midgut of Tenebrio molitor larvae. Insect Biochem. Mol. Biol. 39, 861-874. doi: 10.1016/j.ibmb.2009.10.003

Genta, F. A., Dumont, A. F., Marana, S. R., Terra, W. R., and Ferreira, C. (2007). The interplay of processivity, substrate inhibition and a secondary substrate binding site of an insect exo-beta-1,3-glucanase. Biochim. Biophys. Acta 1774, 1079-1091. doi: 10.1016/j.bbapap.2007.07.006

Genta, F. A., Terra, W. R., and Ferreira, C. (2003). Action pattern, specificity, lytic activities, and physiological role of five digestive $\beta$-glucanases isolated from Periplaneta americana. Insect Biochem. Mol. Biol. 33, 1085-1097. doi: 10.1016/S0965-1748(03)00121-8

Gontijo, N. F., Almeida-Silva, S., Costa, F. F., Mares-Guia, M. L., Williams, P., and Melo, N. (1998). Lutzomyia longipalpis: $\mathrm{pH}$ in the gut, digestive glycosidases, and some speculations upon Leishmania development. Exp. Parasitol. 90, 212-219. doi: 10.1006/expr.1998.4336

Gorin, P. A., and Spencer, J. F. (1968). Structural chemistry of fungal polysaccharides. Adv. Carbohydr. Chem. Biochem. 23, 367-417.

Hall, T. A. (1999). BioEdit: a user-friendly biological sequence alignment editor and analysis program for Windows 95/98/NT. Nucl. Acids. Symp. Ser. 41, 95-98.

Harhay, M. O., Olliaro, P. L., Costa, D. L., and Costa, C. H. (2011). Urban parasitology: visceral leishmaniasis in Brazil. Trends Parasitol. 27, 403-409. doi: 10.1016/j.pt.2011.04.001

Harikrishnan, R., Kim, J. S., Kim, M. C., Balasundaram, C., and Heo, M. S. (2011). Molecular characterization, phylogeny, and expression pattern of ctype lysozyme in kelp grouper, Epinephelus bruneus. Fish Shellfish Immunol. 31, 588-594. doi: 10.1016/j.fsi.2011.06.019

Hashimoto, M., Honda, Y., Nikaidou, N., Fukamizo, T., and Watanabe, T. (2000). Site-directed mutagenesis of Asp280 suggests substrate-assisted catalysis of chitinase A1 from Bacillus circulans WL-12. J. Biosci. Bioeng. 89, 100-102. doi: 10.1016/S1389-1723(00)90031-8

Hughes, A. L. (2012). Evolution of the $\beta$ GRP/GNBP/ $\beta$-1,3-glucanase family of insects. Immunogenetics 64, 549-558. doi: 10.1007/s00251-012-0610-8

Hultmark, D. (1996). Insect lysozymes. EXS 75, 87-102. doi: 10.1007/978-3-03489225-4_6

Ito, Y., Yoshikawa, A., Hotani, T., Fukuda, S., Sugimura, K., and Imoto, T. (1999). Amino acid sequences of lysozymes newly purified from invertebrates imply wide distribution of a novel class in the lysozyme family. Eur. J. Biochem. 259, 456-461. doi: 10.1046/j.1432-1327.1999.00064.x

Jacobson, R. L., and Schlein, Y. (2001). Phlebotomus papatasi and Leishmania major parasites express $\alpha$-amylase and $\alpha$-glucosidase. Acta Tropica 78, 41-49. doi: 10.1016/S0001-706X(00)00164-9

Jollès, P., and Jollès, J., (1984). What's new in lysozyme research? Always a model system, today as yesterday. Mol. Cell. Biochem. 63, 165-189. doi: 10.1007/BF00285225

Julenius, K., Mølgaard, A., Gupta, R., and Brunak, S. (2005). Prediction, conservation analysis and structural characterization of mammalian mucintype O-glycosylation sites. Glycobiol 15, 153-164. doi: 10.1093/glycob/ cwh151

Khajuria, C., Buschman, L. L., Chen, M. S., Muthukrishnan, S., and Zhu, K. Y. (2010). A gut-specific chitinase gene essential for regulation of chitin content of 
peritrophic matrix and growth of Ostrinia nubilalis larvae. Insect Biochem. Mol. Biol. 40, 621-629. doi: 10.1016/j.ibmb.2010.06.003

Kim, M. G., Shin, S. W., Bae, K. S., Kim, S. C., and Park, H. Y. (1998). Molecular cloning of chitinase cDNAs from the silkworm, Bombyx mori and the fall webworm, Hyphantria cunea. Insect Biochem. Mol. Biol. 28, 163-171. doi: 10.1016/S0965-1748(97)00112-4

Kramer, K. J., Corpuz, L., Choi, H. K., and Muthukrishnan, S. (1993). Sequence of a cDNA and expression of the gene encoding epidermal and gut chitinases of Manduca sexta. Insect Biochem. Mol. Biol. 23, 691-701. doi: 10.1016/09651748(93)90043-R

Lee, M. H., Osaki, T., Lee, J. Y., Baek, M. J., Zhang, R., Park, J. W., et al. (2004). Peptidoglycan recognition proteins involved in 1,3-beta-D-glucan-dependent prophenoloxidase activation system of insect. J. Biol. Chem. 279, 3218-3227. doi: 10.1074/jbc.M309821200

Lucena, S. A., Lima, L. S., Cordeiro, L. S., Sant'Anna, C., Constantino, R., Azambuja, P., et al. (2011). High throughput screening of hydrolytic enzymes from termites using a natural substrate derived from sugarcane bagasse. Biotechnol. Biofuels 4:51. doi: 10.1186/1754-6834-4-51

Ma, C., and Kanost, M. R. (2000). A beta1,3-glucan recognition protein from an insect, Manduca sexta, agglutinates microorganisms and activates the phenoloxidase cascade. J. Biol. Chem. 275, 7505-7514. doi: 10.1074/jbc.275. 11.7505

Merzendorfer, H., and Zimoch, L. (2003). Chitin metabolism in insects: structure, function and regulation of chitin synthases and chitinases. J. Exp. Biol. 206(pt 24), 4393-4412. doi: 10.1242/jeb.00709

Moraes, C. S., Lucena, S. A., Moreira, B. H. S., Brazil, R. P., Gontijo, N. F., and Genta, F. A. (2012). Relationship between digestive enzymes and food habit of Lutzomyia longipalpis (Diptera: Psychodidae) larvae: characterization of carbohydrases and digestion of microorganisms. J. Insect Physiol. 58, 1136-1145. doi: 10.1016/j.jinsphys.2012.05.015

Paskewitz, S. M., Li, B., and Kajla, M. K. (2008). Cloning and molecular characterization of two invertebrate-type lysozymes from Anopheles gambiae. Insect Mol. Biol. 17, 217-225. doi: 10.1111/j.1365-2583.2008.00797.x

Pauchet, Y., Freitak, D., Heidel-Fischer, H. M., Heckel, D. G., and Vogel, H. (2009). Immunity or digestion: glucanase activity in a glucan-binding protein family from Lepidoptera. J. Biol. Chem. 284, 2214-2224. doi: 10.1074/jbc. M806204200

Petersen, T. N., Brunak, S., von Heijne, G., and Nielsen, H. (2011). SignalP 4.0: discriminating signal peptides from transmembrane regions. Nat. Meth. 8, 785-786. doi: 10.1038/nmeth.1701

Punta, M., Coggill, P. C., Eberhardt, R. Y., Mistry, J., Tate, J., Boursnell, C., et al. (2012). The Pfam protein families database. Nucleic. Acids Res. 42, D222-D230. doi: 10.1093/nar/gkm960

Ramalho-Ortigão, J. M., Kamhawi, S., Joshit, M. B., Reynoso, D., Lawyer, P. G., Dwyert, D. M., et al. (2005). Characterization of a blood activated chitinolytic system in the midgut of the sand fly vectors Lutzomyia longipalpis and Phlebotomus papatasi. Insect Mol. Biol. 14, 703-712. doi: 10.1111/j.13652583.2005.00601.x

Ramalho-Ortigão, J. M., and Traub-Csekö, Y. M. (2003). Molecular characterization of Llchit1, a midgut chitinase cDNA from the leishmaniasis vector Lutzomyia longipalpis. Insect Biochem. Mol. Biol. 33, 279-287. doi: 10.1016/S0965-1748(02)00209-6

Romero, G. A. S., and Boelaert, M. (2010). Control of visceral leishmaniasis in latin america-a systematic review. PLoS Negl. Trop. Dis. 4:e584. doi: 10.1371/journal.pntd.0000584

Royer, V., Fraichard, S., and Bouhin, H. (2002). A novel putative insect chitinase with multiple catalytic domains: hormonal regulation during metamorphosis. Biochem. J. 366 (pt 3), 921-928. doi: 10.1042/BJ20011764

Royet, J. (2004). Infectious non-self recognition in invertebrates: lessons from Drosophila and other insect models. Mol. Immunol. 41, 1063-1075. doi: 10.1016/j.molimm.2004.06.009

Sant'Anna, M. R., Diaz-Albiter, H., Mubaraki, M., Dillon, R. J., and Bates, P. A. (2009). Inhibition of trypsin expression in Lutzomyia longipalpis using RNAi enhances the survival of Leishmania. Parasi. Vectors 2:62. doi: 10.1186/17563305-2-62

Sant'Anna, M. R. V., Diaz-Albiter, H. M., Aguiar-Martins, K., Cavalcante, R. R., Dillon, V. M., Bates, P. A., et al. (2014). Colonisation resistance in the sand fly gut: Leishmania protects Lutzomyia longipalpis from bacterial infection. Parasit. Vectors. 7:329. doi: 10.1186/1756-3305-7-329
Shen, Z., and Jacobs-Lorena, M. (1997). Characterization of a novel gut-specific chitinase gene from the human malaria vector Anopheles gambiae. J. Biol. Chem. 272, 28895-28900. doi: 10.1074/jbc.272.46.28895

Sietsma, J. H., and Wessels, J. G. (1981). Solubility of (1 leads to 3)-beta-D/(1 leads to 6)-beta D-glucan in fungal walls: importance of presumed linkage between glucan and chitin. J. Gen. Microbiol. 125, 209-212.

Soares, R. P. P., and Turco, S. J. (2003). Lutzomyia longipalpis (Diptera: Psychodidae: Phlebotominae): a review. An. Acad. Bras. Cienc. 75, 301-330. doi: 10.1590/S0001-37652003000300005

Souza-Neto, J. A., Gusmão, D. S., and Lemos, F. J. (2003). Chitinolytic activities in the gut of Aedes aegypti (Diptera: Culicidae) larvae and their role in digestion of chitin-rich structures. Comp. Biochem. Physiol. A Mol. Integr. Physiol. 136, 717-724. doi: 10.1016/S1095-6433(03)00224-1

St. Pierre, S. E., Ponting, L., Stefancsik, R., and McQuilton, P. (2014). The FlyBase. FlyBase 102 - advanced approaches to interrogating FlyBase. Nucleic Acids Res. 42, D780-D788. doi: 10.1093/nar/gkt1092

Sun, Z., Wu, W., and Zhang, G. (2011). Structure and expression of $\beta$-1,3-glucan recognition proteins from the ghost moth, Thitarodes pui (Hepialidae), and their response to Beauveria bassiana infection. J. Insect Physiol. 57, 1660-1669. doi: 10.1016/j.jinsphys.2011.08.019

Tamura, K., Peterson, D., Peterson, N., Stecher, G., Nei, M., and Kumar, S. (2011). MEGA5: molecular evolutionary genetics analysis using maximum likelihood, evolutionary distance, and maximum parsimony methods. Mol. Biol. Evol. 28, 2731-2739. doi: 10.1093/molbev/msr121

Tanahashi, M., Matsushita, N., and Togashi, K. (2009). Are stag beetles fungivorous? J. Insect Physiol. 55, 983-988. doi: 10.1016/j.jinsphys.2009.07.002

Telleria, E. L., Araújo, A. P. O., Secundino, N. F., d'Avila-Levy, C. M., and TraubCsekö, Y. M. (2010). Trypsin-like serine proteases in Lutzomyia longipalpis expression, activity and possible modulation by Leishmania infantum chagasi. PLoS ONE 5:e10697. doi: 10.1371/journal.pone.0010697

Telleria, E. L., Pitaluga, A. N., Ortigao-Farias, J. R., de Araujo, A. P. O., RamalhoOrtigao, J. M., and Traub-Cseko, Y. M. (2007). Constitutive and blood mealinduced trypsin genes in Lutzomyia longipalpis. Archiv. Insect Biochem. Physiol. 66, 53-63. doi: 10.1002/arch.20198

Telleria, E. L., Sant'Anna, M. R., Alkurbi, M. O., Pitaluga, A. N., Dillon, R. J., and Traub-Csekö, Y. M. (2013). Bacterial feeding, Leishmania infection and distinct infection routes induce differential defensin expression in Lutzomyia longipalpis. Parasit. Vectors 6:12. doi: 10.1186/1756-3305-6-12

Terra, W. R., and Ferreira, C. (1994). Insect digestive enzymes - properties, compartmentalizationand function. Comp. Biochem. Physiol. 109B, 1-62.

Terra, W. R., and Ferreira, C. (2005). "Biochemistry of digestion" in Comprehensive Molecular Insect Science, Vol. 4: Biochemistry and Molecular Biology, eds L. I. Gilbert, K. Iatrou, and S. S. Gill (Amsterdam: Elsevier), 171-224.

Tsai, Y. L., Chang, C. C., Chuang, S. T., and Chomel, B. B. (2011). Bartonella species and their ectoparasites: selective host adaptation or strain selection between the vector and the mammalian host? Comp. Immunol. Microbiol. Infect. Dis. 34, 299-314. doi: 10.1016/j.cimid.2011.04.005

Venancio, T. M., Cristofoletti, P. T., Ferreira, C., Verjovski-Almeida, S., and Terra, W. R. (2009). The Aedes aegypti larval transcriptome: a comparative perspective with emphasis on trypsins and the domain structure of peritrophins. Insect Mol. Biol. 18, 33-44. doi: 10.1111/j.1365-2583.2008.00845.x

Volf, P., Kiewegová, A., and Nemec, A. (2002). Bacterial colonization in the gut of Phlebotomus duboscqi (Diptera: Psychodidae): transtadial passage and the role of female diet. Folia Parasitol. 49, 73-77. doi: 10.14411/fp.2002.014

Wang, X., Fuchs, J. F., Infanger, L. C., Rocheleau, T. A., Hillyer, J. F., Chen, C. C., et al. (2005). Mosquito innate immunity: involvement of beta 1,3-glucan recognition protein in melanotic encapsulation immune responses in Armigeres subalbatus. Mol. Biochem. Parasitol. 139, 65-73. doi: 10.1016/j.molbiopara. 2004.09.009

Wermelinger, E. D., and Zanuncio, J. (2001). Development of Lutzomyia intermedia and Lutzomyia longipalpis (Diptera: Psychodidae: Phlebotominae) larvae in different diets. Brazil. J. Biol. 61, 405-408. doi: 10.1590/S1519-69842001000 300009

WHO. (2014). Leishmaniasis. Available online at: http://www.who.int/ leishmaniasis/en/

Young, D. G., Perking, P. V., and Endris, R. G. (1981). A larval diet for rearing phlebotominae sand flies (Diptera: Psychodidae). J. Med. Entomol. 18:446.

Zavalova, L. L., Baskova, I. P., Lukyanov, S. A., Sass, A. V., Snezhkov, E. V., Akopov, S. B., et al. (2000). Destabilase from the medicinal leech is a representative of 
a novel family of lysozymes. Biochem. Biophys. Acta 1478, 69-77. doi: 10.1016/ S0167-4838(00)00006-6

Zhang, J., Zhang, X., Arakane, Y., Muthukrishnan, S., Kramer, K. J., Ma, E., et al. (2011). Comparative genomic analysis of chitinase and chitinase-like genes in the African malaria mosquito (Anopheles gambiae). PLoS ONE 6:e19899. doi: 10.1371/journal.pone.0019899

Zhu, Q., Arakane, Y., Beeman, R. W., Kramer, K. J., and Muthukrishnan, S. (2008). Characterization of recombinant chitinase-like proteins of Drosophila melanogaster and Tribolium castaneum. Insect Biochem. Mol. Biol. 38, 467-477. doi: 10.1016/j.ibmb.2007.06.011

Conflict of Interest Statement: The authors declare that the research was conducted in the absence of any commercial or financial relationships that could be construed as a potential conflict of interest.
Received: 12 June 2014; accepted: 07 July 2014; published online: 05 August 2014. Citation: Moraes CS, Diaz-Albiter HM, Faria MV, Sant'Anna MRV, Dillon RJ and Genta FA (2014) Expression pattern of glycoside hydrolase genes in Lutzomyia longipalpis reveals key enzymes involved in larval digestion. Front. Physiol. 5:276. doi: 10.3389/fphys.2014.00276

This article was submitted to Integrative Physiology, a section of the journal Frontiers in Physiology.

Copyright (C) 2014 Moraes, Diaz-Albiter, Faria, Sant'Anna, Dillon and Genta. This is an open-access article distributed under the terms of the Creative Commons Attribution License (CC BY). The use, distribution or reproduction in other forums is permitted, provided the original author(s) or licensor are credited and that the original publication in this journal is cited, in accordance with accepted academic practice. No use, distribution or reproduction is permitted which does not comply with these terms. 